\title{
Intracellular Notch1 May Induce a Conformational Change in CSL/DNA, without Forming ICN1/CSL/DNA Molecular Complex, in Vitro
}

\author{
Alexei A. Stortchevoi* \\ Department of Pathology, Yale University, New Haven, USA \\ Email: alexei.stortchevoi@yale.edu
}

Received November 25, 2012; revised December 26, 2012; accepted January 10, 2013

Copyright (C) 2013 Alexei A. Stortchevoi. This is an open access article distributed under the Creative Commons Attribution License, which permits unrestricted use, distribution, and reproduction in any medium, provided the original work is properly cited.

\begin{abstract}
Intracellular Notch (ICN) initiates DNA transcription in cooperation with CSL that acts as repressor in the absence of ICN. The ICN mediates recruitment of MAML protein, leading to the formation of minimal transcriptional complex, MAML/ICN/CSL/DNA. Crystal structure reveals that different conformations exist between the free (CSL/DNA) and bound (ICN/MAML/CSL/DNA) forms. The significance of this modulation of the CSL/DNA molecular complex can be better understood by experimental approaches that aim to elucidate the cause and timing of these events. There are four orthologues of human ICN (ICN1-4). We studied interactions between human full-length ICN1 and CSL/DNA without involvement of MAML, in vitro, and found that 1) the EMSA profile of CSL/DNA is altered in the presence of ICN1 as a consequence of an intrinsic change(s) in CSL/DNA, and not due to the formation of an ICN/CSL/DNA molecular complex; 2) ICN1 destabilizes CSL/DNA. These findings indicate that human ICN1 functions to modulate the CSL/DNA molecular complex for subsequent recruitment of MAML, and that modulated CSL/DNA cannot accommodate ICN1 in the absence of MAML. The latter in turn, implies that the formation of the MAML/ICN1/CSL/DNA is likely to be a collective event, wherein preassembly of MAML and ICN1 as a binary complex co-localizes at the CSL/DNA promoter site, or the MAML/ICN1/CSL complex is pre-assembled prior to binding to the promoter, rather than ICN1 arriving at CSL/DNA ahead of MAML and/or other associated transcription factors. The novel finding that ICN1 destabilizes the CSL/DNA complex opens new possibilities of transcriptional regulation by Notch.
\end{abstract}

Keywords: CBF1; CSL; Hes1 Promoter; Notch; RbpJ; Su(H)

\section{Introduction}

Notch is a transmembrane receptor protein that controls numerous developmental decisions through binding of ligands displayed on the surface of adjacent cells. Notch signaling pathway regulates cell fate decisions in neurogenesis [1], T cell development [2] and hypoxia [3] and has been implicated in multiple cross talks with major pathways that influence cell proliferation, differentiation, survival and migration [4]. Aberrations in Notch receptors have been linked to malignancies [5-9], and lately, the components of Notch signal transduction have been targeted in anti-cancer drug development [10-12].

Upon ligand binding, the Notch receptor is proteolytically cleaved at the membrane site, and intracellular

*Current address: Department of Genetics, Harvard Medical School, Center for Human Genetic Research, Massachusetts General Hospital's Richard B. Simches Research Center, Boston, USA.
Notch (ICN) migrates to the nucleus, where it interacts with various promoters to stimulate transcription in cooperation with transcription factor CSL (also known as RBP-J, CBF1, Su(H) and LAG-1), reviewed [13-15]. In the absence of ICN, CSL acts as a transcriptional repressor, bound at the promoter to "GTGGGAAA" CSL specific sequence. The ICN has not been found to bind directly to DNA but its RAM domain (RAM = RBP-J Associating Molecule) and Ankyrin domain (ANK) interact with CSL [16]. Transcriptional repression by CSL is reportedly achieved through recruitment of histone deacetylase complexes, SMRT/mSin3A/HDAC-1 or NCor/ nSin3A/HDAC-1 [17], CIR/SAP30/HADC-2 [18] or SHARP/CtIP [19] to a gene promoter site and compacting of the chromatin. It is believed that ICN1 plays an important role in displacing the co-repressor complexes, allowing the chromatinized DNA to unfold and thus fa- 
cilitating transcription. This ICN function is supported by Mastermind-like, a human homologue of Drosophila protein (MAML) [20-22], Ski-interacting protein (SKIP) that binds both, CSL and ICN [23] and histone acetylase $\mathrm{CBP} / \mathrm{p} 300$ [20]. MAML is also thought to control withdrawal of ICN1 from the transcription complex [20,24]. Other proteins involved in ICN-mediated transcription include negative regulator KyoT2 [25], transcriptional co-activator p300 [26], hypoxia-inducible fator 1 alfa (Hif1a) $[3,27]$, mediator protein Med220, cell-cycle depended kinase 7 (CDK7) and CDK8 [28], elongation factors FACT, Spt6, P-TEFb, reviewed [13]. While the number of transcription factors involved is extensive, and continues to grow, the sequence of the events leading to transcription and its turnover is far from been clearly understood.

One of the lingering questions surrounding ICN mediated transcription events is whether ICN binds independently to CSL/DNA, or is dependent upon other factors to form the ICN/CSL/DNA complex. Some data point to a higher probability of an assisted rather than independent ICN1 binding to CSL/DNA in mammals: Timed ChIP/PCR study shows that the arrival and the departure of ICN1 at the CSL-occupied HES1 promoter occurs within the same $30 \mathrm{~min}$. interval that is associated with MAML1 and SKIP binding to CSL/DNA [28]. Crystal structure of the CSL/HES1 promoter fragment with the ANK domain of murine ICN1 and N-terminal peptide of MAML1 shows that mutual dependence of these proteins is required to maintain stability of the association [29]. When crystal structures are compared between CSL/DNA [30] and MAML/ICN1/CSL/DNA (harboring the RAMANK domain of mouse ICN1) [31], it reveals conformational change occurs in CSL/DNA; this observation raises the important question of whether the change in CSL/DNA structure is induced by ICN1 only, or via a synergistic interaction between ICN1 and MAML.

Human ICN1 is $\sim 90 \%$ homologous to the mouse counterpart, with most of the discrepancies located within the C-terminal "Trans-Activation Domain" that is absent in the crystal structures, leaving the impact of interspecies differences open to speculation.

We analyzed the impact of full-length human recombinant ICN1 on CSL/DNA binding. The study was designed to include only the initial trio: human HES1 promoter DNA, full-length human CSL and full-length human ICN1. We show that ICN1 does not form stable association with CSL/DNA in the absence of MAML (at the conditions used) but induces a change in the CSL/ DNA molecular complex that alters its electromobility in EMSA. Another effect of the ICN1 is that it destabilizes the bond between the CSL protein and Hes 1 promoter, and that the degree of CSL dissociation is dependent on the length of DNA fragment that is used for the EMSA.
The results of these experiments suggest that human ICN1 can induce a conformational change(s) in CSL/ DNA, and recruit additional DNA sequence(s) that maintain CSL binding to the core promoter during the interaction. Our data complement and expand the existing knowledge of ICN1 interaction with CSL/DNA; we demonstrate that use of the full-length human ICN1 (and not the RAMANK truncated form of murine ICN), is capable of conformational change(s) in CSL/DNA. In addition, we show that ICN1 alone is capable of inducing the change (likely, to accommodate MAML binding to CSL/DNA) but cannot form stable association with CSL/DNA without MAML (likely because the new conformation of CSL/DNA favors binding of ICN1/MAML but not ICN1). The results imply that in human species binding of MAML and ICN1 to CSL/DNA at Hes1 promoter is a simultaneous, mutually assisted event. Another interesting finding of this work is destabilization of the CSL/DNA complex by Notch, which potentially may play a role in negative regulation of transcription.

\section{Materials and Methods}

\subsection{Antibodies and Transfection Reagents}

Anti-Flag M2 antibody (F1804), anti-Flag M2 antibody HRP conjugate (A8592), anti-Flag M2 affinity gel (A2220), anti-HA monoclonal antibody, clone HA-7, HRP conjugate (H6533), anti-HA monoclonal antibody (H9658), anti-polyHistidine monoclonal antibody (H1029) were obtained from Sigma (Sigma Chemicals, St. Louis, MO, USA); anti-6xHis tag antibody HRP conjugate (ab1187) was obtained from Abcam (Abcam, Cambridge, UK), anti-ICN1 polyclonal serum (goat) was prepared in the lab. DMEM and RPMI media, Lipofectamine 2000 and Lipofectamine TM were purchased from Invitrogen (Invitrogen, Carisbad, CA, USA).

\subsection{DNA Sequences}

For EMSA probes, we used fragments of HES1 promoter (ENSEMBL genome ID: ENST00000232424, human chromosome 3: $195,853,833$ to $195,853,892)$. The basic 59 bp probe imitated -101 to -42 fragment of the transcription start $(-337$ to -278 before the ATG translation initiation codon) of that sequence. The purified DNA duplexes were purchased from Eurofins MWG (Huntsville, AL, USA, oligo-us@eurofins.com).

\subsection{CSL and ICN1 Protein Expression in E. coli}

Recombinant ICN1 and CSL genes and cloning sites can be viewed in the supplement to this article.

The genes were cloned in pET28a vector (Novagen, Madison, WI, USA) and expressed in BL21.DE3 strain 
of E. coli. Expression of the ICN1 and 6His-ICN1-6His recombinant proteins: $0.4 \mathrm{mM}$ IPTG at OD600 $=0.8,3$ hours, $37^{\circ} \mathrm{C}$ after induction; Expression of CSL, CSL6His Flag-CSL and Flag-CSL-6His recombinant proteins: $0.4 \mathrm{mM}$ IPTG at OD600 $=0.8$ followed by overnight growth at $16^{\circ} \mathrm{C}$ : at such conditions $\sim 80 \%$ of the recombinant CSL protein was soluble. The 6His- tagged proteins were purified using Ni-NTA Agarose (Invitrogen R901-01 or Qiagen 30230) according to manufacturers' instructions at native conditions. After purification, proteins were dialyzed in phosphate buffer $(50 \mathrm{mM}$ $\mathrm{NaH}_{2} \mathrm{PO}_{4} \mathrm{pH} 8.0,500 \mathrm{mM} \mathrm{NaCl}$ ) snap-frozen and stored at $-80^{\circ} \mathrm{C}$ or stored at $-20^{\circ} \mathrm{C}$ in if dialyzed in phosphate buffer in presence of $40 \%$ glycerol.

\subsection{CSL and ICN1 Protein Expression in HEK293T Cells}

HEK293T cells were transiently transfected in a $10 \mathrm{~cm}$ tissue culture dish with $2-10 \mu \mathrm{g}$ of a recombinant vector DNA, using Lipofectamin 2000 or Lipofectamin TM reagents. Cells were harvested for protein extract preparation in 24 - 72 hours: washed with an ice-cold PBS, then scraped from the plate and transferred to a $15 \mathrm{ml}$ Falcon polypropylene tube. After pelleting by centrifugation, the cells were lysed by osmotic shock on ice for 10 min. in $1 \mathrm{ml} / 10^{7}$ cells of the Hypotonic lysis buffer $(10$ $\mathrm{mM}$ TrisHCl, pH 7.4, $10 \mathrm{mM} \mathrm{NaCl}, 2 \mathrm{mM}$ EDTA, 0.5\% Triton X-100) in the presence of $1 \mathrm{mM}$ PMSF and a 1:50 dilution of protease inhibitor cocktail (Sigma P8340). After lysis, the $\mathrm{NaCl}$ concentration was adjusted to 150 $\mathrm{mM}$ and the cell extract was cleared of debris by centrifugation at $4^{\circ} \mathrm{C}$ for 15 minutes at maximum speed on a microcentrifuge, the extracts were taken for EMSA analysis immediately or snap-frozen in aliquots and stored at $-80^{\circ} \mathrm{C}$ prior to single use. Protein expression in the extracts was confirmed by Western blotting.

\subsection{ChIP/PCR Assay}

Chromatin immunoprecipitation was adapted from the published protocol [32] with adjustment for antibodyagarose conjugates (precipitation with protein A was not necessary). Small fractions of cell culture were scraped prior to cross-linking with formaldehyde for analysis of protein expression by SDS-PAGE/Western blotting. PCR Primers: CCCTGGCTCCAAAAGAAATAGAC and GAAGTTTCACACGAGCCGTTCG. After running PCR products in $1.5 \%$ TAE agarose gel, quantification of the bands was done with GeneTools Version 4.01 software (Syngene (A Division of Synoptics Ltd), Beacon House, Cambridge, CB41TF, England).

\subsection{EMSA}

The EMSA Accessory Kit (Novagen 71282-3) was used for sample preparation according to manufacturer's instructions. ( $4 \times$ EMSA binding buffer $=400 \mathrm{mM} \mathrm{KCl}, 8$ mM HEPES, 2 mM DTT, 0.8 mM EDTA, 80\% glycerol, pH $8.0 ; 1 \times$ EMSA binding buffer was supplemented with sonicated salmon sperm DNA (125 - $200 \mathrm{ng}$ ) and Poly $(\mathrm{dI}-\mathrm{dC})(\mathrm{dI}-\mathrm{dC})(0.01 \mathrm{U}))$. EMSA sample $(20 \mathrm{ml})$ contained, unless specified otherwise, $300 \mathrm{nM}$ of DNA probe (10,000 - 100,000 cpm of ${ }^{32} \mathrm{P}$ activity) $2-10 \mathrm{mg}$ of total protein extract or $100-400 \mathrm{nM}(100-300 \mathrm{ng}) / \mathrm{re}-$ action of purified CSL or ICN1. The DNA/CSL mix was pre-incubated on ice for 10 minutes prior to loading in gel. After addition of ICN1, the sample was loaded in gel and ran immediately or pre-incubated on ice for up to 30 minutes, and then ran, at $7.5 \mathrm{~V} / \mathrm{cm}$ for $2-8$ hours (depending on the required resolution) at room temperature. The $15 \times 20 \mathrm{~cm} 1 \mathrm{~mm}-1.5 \mathrm{~mm}$ thick $4 \%-6 \%$ TBE acrylamide:bis (29:1) gel was pre-run for $30-40 \mathrm{~min}$ prior to loading the samples. After the run was finished, the gel was taken off the glass plates, wrapped in plastic film and exposed to autoradiography film or to a phosphorimaging screen.

\subsection{EMSA/Western Blotting}

The DNA and proteins from EMSA gel were transferred on to a PVDF membrane (Immobilon-P Cat. No: IPVH 00010 Millipore, Billerica, MA, USA), using the optimized protocol for semi-dry transfer: Three transfer buffers, containing $10 \%$ methanol were: anode buffer I $(0.3$ $\mathrm{M}$ Tris $\mathrm{pH} 10.4)$, anode buffer II (25 mM Tris $\mathrm{pH} 10.4)$, and cathode buffer $(25 \mathrm{mM}$ Tris and $40 \mathrm{mM}$ e-aminocaproic acid, $\mathrm{pH}$ 9.4). The transfer membrane, sandwiched between transparent plastic sheets, was then exposed to a film ( 2 hours at $-80^{\circ} \mathrm{C}$ with enhancer screen), or a phosphorimaging screen, with reference points marked at the membrane. After radioactivity exposure, Western blotting analysis was done in a single step, using an HRP-conjugated (anti-Flag, anti-HA or anti-6His) antibody.

\subsection{Phosphorimaging Analysis}

Special care was taken to ensure equal distribution of CSL and ${ }^{32}$ P-labeled DNA probe between the "No ICN1" and "+ICN1" samples prior to loading. After each run, the wet EMSA gel was placed between plastic sheets and exposed to ${ }^{32} \mathrm{P}$ sensitized phosphorimaging screen for 2 6 hours. Image reading and quantification was done with Image Quant software (Molecular Dynamics, Foster City, CA).

\subsection{DNase Footprinting Analysis}

$20 \mu \mathrm{l}$ of EMSA sample in EMSA buffer containing $10,000-20,000 \mathrm{cpm}{ }^{32} \mathrm{P}$-labeled DNA probe was treated with 1 - $10 \mathrm{u}$ of DNase I for 5 - 10 seconds prior to ex- 
traction once with phenol (volume adjusted to $100 \mu \mathrm{l}$ ), once-with phenol/chloroform, and once-with chloroform. DNA was ethanol precipitated with added $4 \mu \mathrm{g}$ glycogen (as carrier). Pellets were re-dissolved in $20 \mu \mathrm{l}$ of sequencing buffer and $2-4 \mu \mathrm{l}$ per lane were taken for analysis in a sequencing gel.

\section{Results}

\subsection{Testing of Recombinant CSL and ICN1 in EMSA Shows That ICN1 Has Supershifting and Weakening Effects on CSL/DNA Band}

DNA probes for Electromobility Shift Assay (EMSA), used in this work, represented the fragments of human HES1 promoter, where it contains two consensus CSL binding sites [33]. The 59 bp wild type (WT) probe, "59 bp CPS", referred to as CPS for CSL Paired Sites [34] had the two original sites: one site - GTGGGAAA — was closer to 5'-end of the fragment and designated " 5 ' $\mathrm{CS}$ " (CS = CSL Site)," and the other-TTTCACAC (antiparallel) - was closer to 3'-end of the fragment and designated "3'CS". Derived from the "59 bp CPS" probe were two shorter WT probes: "18 bp 5'CS" contained only one (5'CS) CSL site [29] and "21 bp No CS" contained no CSL sites. There were also three full-length mutant probes: "59 bp 5'CS" and "59 bp 3'CS" had only one alternative CSL site-active and another-inactivated by mutations, and "59 bp No CS", where both sites were inactivated (Figure 1(a)).

Using the WT and the mutant 59 bp probes, we confirmed CSL site-specificity of DNA shifts in EMSA with HEK293T cell extract overexpressing untagged fulllength recombinant human CSL (Figure 1(b), right panel), and titrated the amount per reaction of bacterially expressed purified CSL-6His to comparable results (Figure 1(b), left panel). Consistent with the number of active CSL sites per probe, "59 bp CPS" produced two bands in the shift, corresponding to one and two CSL molecules bound to a single DNA probe (Figure 1(b) lane 1); "59 bp 5'CS" and "59 bp 3'CS" each produced single band (Figure 1(b) lanes 2 and 4); and "59 bp No CS"-produced no EMSA shifts at all (Figure 1(b) lane 3). Having confirmed experimentally that the mutations eliminated CSL specificities of the CSL sites, we no longer used "59 bp No CS" probe as negative control but used the "21 bp No CS", instead.

After confirming the binding of the recombinant CSL to DNA in EMSA, we tested "ICN1+DNA" mixtures. The ICN1 produced no specific EMSA shifts with any of the CS-containing probes (not shown) but when added to pre-incubated CSL/DNA, it created visible impacts: 1) the primary CSL/DNA shift was retarded (Figure 1(c) lane 2) and 2) the shift was either weakened (as with "59
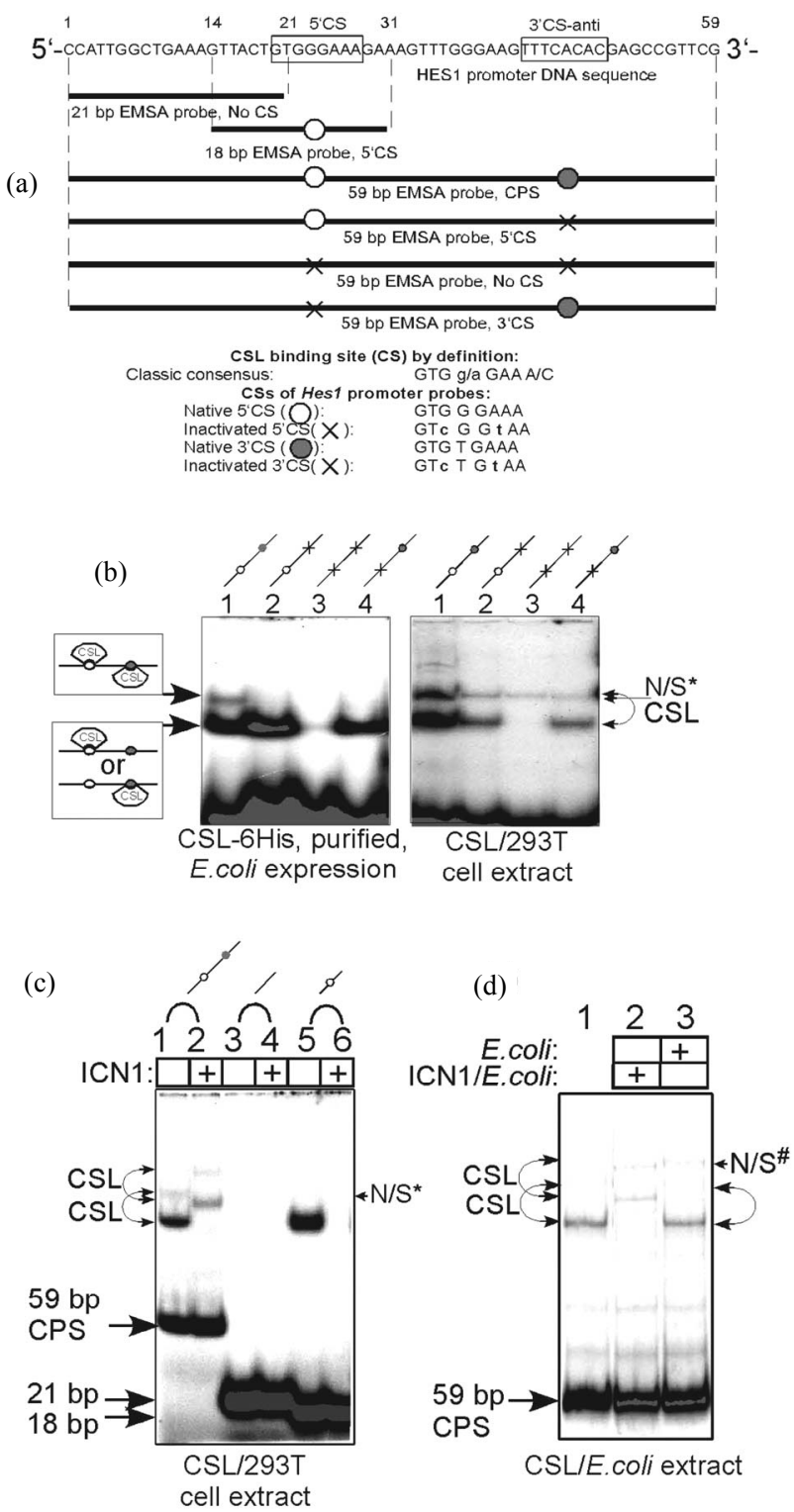

Figure 1. Initial EMSA with Hes1 promoter probes. (a) Schematic EMSA probes are linked by dotted lines with the 59 bp reference sequence. The CSL binding sites (CSs)boxed in the sequence-are depicted as circular shapes in the diagram: Clear-for active 5'CS; filled-for active 3'CS. Inactivated CSs are depicted as X-shapes instead of circular. The original $C S$ sequences and the inactivating mutations (substituted nucleotides-in small caps) are shown below, referred to consensus sequence [33]; (b) Testing the 59 bp probes (shown schematically above the EMSA lanes) with $100 \mathrm{nM}$ purified CSL (left panel) and with $8 \mathrm{mg}$ of CSL/ 293T extract (right panel). Diagram on the left of EMSA picture depicts putative CSL/DNA complexes. N/S*-"Nonspecific” DNA-binding activity of 293T extract; (c) EMSA of "DNA + $8 \mathrm{mg}$ of CSL/293T extract" and "DNA/8 mg of CSL/293T extract $+200 \mathrm{nM}$ of 6His-ICN1-6His" sample pairs with different DNA probes, depicted above; (d) EMSA of $59 \mathrm{bp}$ CPS probe with soluble bacterial extracts $(5 \mathrm{mg})$ expressing untagged CSL and ICN1. N/S ${ }^{\#-N o n-s p e c i f i c ~}$ DNA-binding activity of E.coli BL21.DE3 extract. 
bp CPS" in Figure 1(c) lane 2) or completely disap peared (as with "18 bp 5'CS" in Figure 1(c) lane 6). To exclude artifact, potentially brought by 6 Histag of recombinant ICN1, we replaced the purified 6His-ICN1$6 \mathrm{His}$ in the reaction by soluble E. coli extract, overexpressing untagged ICN1 (ICN1/E.coli): the "ICN1/ E.coli" extract produced the same effects as the purified 6His-ICN1-6His protein (Figure 1(d) lane 2) whereas E.coli extract without ICN1 expression (E. coli) had no impact (Figure 1(d) lane 3).

\subsection{Supershifting of CSL/DNA Band in EMSA Is Not Caused by Formation of ICN1/CSL/DNA Complex, Because ICN1 Is Absent from the Shift}

The retardation of primary EMSA shift in "+ICN1" sample could reflect the formation of ICN1/CSL/DNA molecular complex with lower electromobility mobility due to its larger size than CSL/DNA, but could also be a consequence of an allosteric modification of CSL/DNA by ICN1, where modified form of CSL/DNA would have lower electromobility in EMSA. To find out what was really the case, we took three approaches to detect whether ICN1 was present in the secondary shift.

Firstly, we tried supershifting with monoclonal antiHis and polyclonal anti-ICN1 antibodies. Both antibodies were proven in Western blotting but neither of them produced any effect on the secondary CSL/DNA shift in EMSA (Figure 2(a) lanes 5 and 7), which would be the expected if ICN1 formed the ICN1/CSL/DNA molecular complex. At the same time, supershifting worked efficiently with anti-Flag antibody, confirming the presence of Flag-CSL in the primary and the secondary shifts (Figure 2(a) lanes 3 and 6).

Secondly, we used sequentially decreasing concentrations of ICN1 with constant concentration of CSL, in EMSA reactions. The expected outcome was coexistence in EMSA of two bands (one for CSL/DNA and one for ICN1/CSL/DNA) when CSL was in excess to ICN1 and the amount of ICN1 was sufficient to produce a visible secondary shift. Instead, only the secondary shift was present and no primary shifts, with four sequential dilutions of ICN1 (Figure 2(b) lanes 2 - 5); The last sample in the range, where ICN1 concentration was the lowest, contained only the primary shift but no secondary shifts (Figure 2 lane 6), presumably because the dilution of ICN1 reached the point where supershifting effect could no longer be produced at given conditions. Such result was more consistent with a conformational change in the CSL/DNA molecular complex than with ICN1 joining the complex.

Finally, we used EMSA/Western blotting technique to

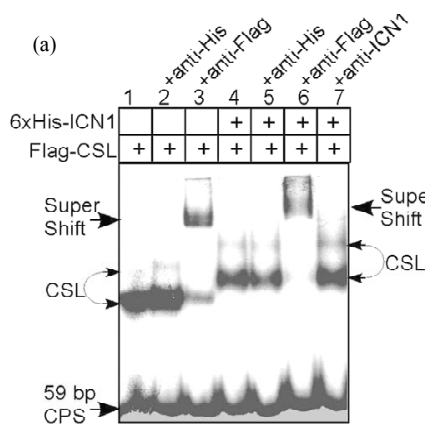

(b)
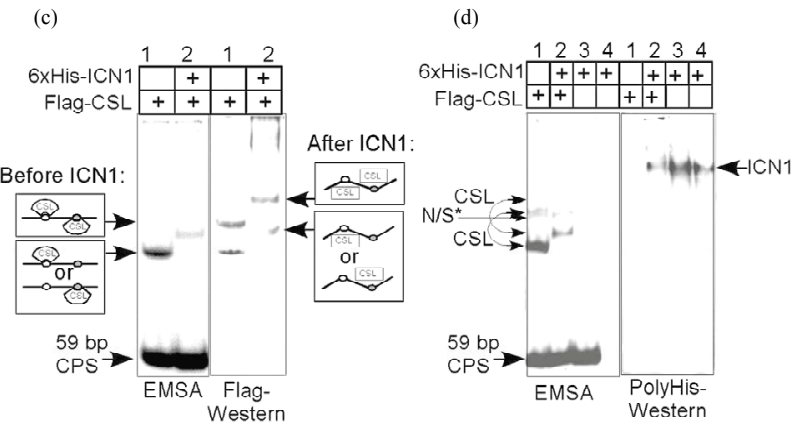

Figure 2. Analysis of EMSA bands. (a) Primary and secondary EMSA shifts were supershifted with $100 \mathrm{ng}$ anti-Flag monoclonal antibody (lanes 3 and 6), but not with either100 ng anti-6×His monoclonal antibody (lanes 2 and 5) or 100 ng anti-ICN1 polyclonal serum (lane 7). The samples contained $300 \mathrm{nM}$ of ${ }^{5}$ '-end-labeled $\left[{ }^{32} \mathrm{P}\right] 59$ bp CPS DNA probe, $100 \mathrm{nM}$ purified Flag-CSL and $200 \mathrm{nM}$ purified 6HisICN1-6His; (b) Titration of decreasing concentrations of ICN1 fails to induce a co-existance of "CSL/DNA" and "ICN1/CSL/DNA" EMSA bands (ICN1: lane 1 = 0 nM; lane 2 = $200 \mathrm{nM}$; lane 3 = $150 \mathrm{nM}$; lane 4 = $100 \mathrm{nM}$; lane 5 = 50 $\mathrm{nM}$; lane 6 = $10 \mathrm{nM}$. CSL: $100 \mathrm{nM}$; DNA: $300 \mathrm{nM}$; (c) Flag-CSL was detected by Western blotting (right panel) in primary and secondary (with ICN1) shifts. The diagrams at the sides of the picture panels show the putative CSL/DNA molecular complexes that form the shifts; (d) The recombinant $6 \times$ His- ICN1-6xHis protein was detected by anti-His Western blotting (right panel) not in the DNA shift but much higher in the gel,. The 6His-ICN1-6His protein (200 nM) was present in the samples 2 - 4, including "No DNA" in lane 4. N/S* in lanes 1 and 2-“Non-specific" DNA-binding activity of $293 \mathrm{~T}$ extract, located in gel very closely to 2 XCSL/DNA band.

establish the exact locations of 6His-ICN1-6His and FlagCSL proteins in EMSA gel. Once again, the presence of Flag-CSL in the primary and the secondary shifts was confirmed (Figure 2(c) right panel, lanes 1 and 2), however 6His-ICN1-6His was not in the shift but detected at a much higher position in the gel, not co-localizing with DNA-bound CSL (Figure 2(d) right panel, lanes 2 - 4).

Based on these findings we concluded that the secondary EMSA shift of "+ICN1" sample was not ICN1/ CSL/DNA molecular complex but an alternative conformation of CSL/DNA molecular complex, induced by ICN1. 


\subsection{Weakening of EMSA Shifts in "+ICN1" Samples is Due to CSL/DNA Dissociation}

The second effect, weakening of EMSA shift in "+ICN1" samples, indicated dissociation of DNA from CSL/DNA molecular complex. We measured the effect quantitatively and confirmed that when the amount of DNA in the shift was decreasing, the amount of free (unbound) DNA was complementary increasing in the same sample (Figure 3(a) The total DNA loads of "-ICN1" and "+ICN1" samples varied negligibly (raw measurements prior to converting to "\% of total" are provided in Table S1), excluding loss of DNA by degradation. The CSL/ DNA was dissociated about $50 \%$ when 59 bp CPS probe was used (Figure 3(a) and almost complete when the 18 bp 5'CS probe was used.

Since the two probes had different length (59 bp vs 18 bp) and different number of CSL sites per probe $\left(5^{\prime} \mathrm{CS}+\right.$ $3^{\prime} \mathrm{CS}$ vs $5^{\prime} \mathrm{CS}$ only), we investigated what had greater influence on the extent of CSL/DNA dissociation in presence of ICN1, and found that it was the length of the probe. In Figure 3(b), EMSA was performed alongside for $59 \mathrm{bp} \mathrm{3'CS,} 59 \mathrm{bp} 5^{\text {' }} \mathrm{CS}$ (each having one but alternative CS) and 59 bp CPS (having two CSs). All three probes shared the same sequence (except CSL sites) and equal length (59 bp): the dissociating/retardation effect in presence ICN1 was similar for all three probes (Figure 3(b) lanes 2, 4 and 8). However between the 18 bp 5'CS and the $59 \mathrm{bp} \mathrm{5'CS}$ probes, having the same CSL binding site but different length, the difference was sharp (Figure 3(b) lane 12 vs 14).

\subsection{DNase Footprinting Analysis Supports Conformational Change in DNA/CSL in Presence of ICN1}

Since the length of the DNA probe played a role in the extent of DNA dissociation from CSL/DNA in presence of ICN1 whereas CSL binding sites did not seem to have influence, we were seeking an additional evidence of involvement of sequence(s) other than CSL binding sites of the " 59 bp CPS" during the DNA/CSL-ICN1 interaction. Our method of choice was DNase footprinting analysis. We kept the CSL concentration at minimum (to enhance the sensitivity to ICN1) and added ICN1, trying to identify the changes in the degenerate DNA ladder. The gel image in left panel of Figure 3(c) shows titration of CSL protein with $59 \mathrm{bp}$ CPS, in footprinting (lanes 4 8): the footprints left by CSL in DNA degenerate ladder are indicated by arrows on the left and the vertical bars at the right side of the picture. In the right panel of Figure 3(c), $2 \mathrm{mg}$ and $4 \mathrm{mg}$ of 6His-ICN1-6His (lanes 4 and 5) were added to $1 \mathrm{mg}$ of CSL-6His (lanes 3-5) compared to controls where soluble E.coli extract, not expressing CSL or ICN1 was used in place of CSL (lanes 6-8) and where 6His-ICN1-6His was used alone (lane 9). As seen from the figure, the "CSL + ICN1" samples (lanes 4 and 5) showed difference from all other samples at positions 34 - 36 (TTG) of the degenerate DNA ladder, implicating a structural change, consistent with EMSA results.

\subsection{The Results of ChIP/PCR of Hes1 Promoter from HEK293T Cells, Transfected with CSL and ICN1 Genes Are Consistent with Partial CSL/DNA Dissociation in Presence of Overexpressed ICN1 inside the Cell}

In the previous section, we showed that full-length human ICN1 caused partial dissociation of CSL from the Hes 1 promoter in vitro in the absence of stabilizing transcription factors. To find out if similar effect of ICN1 takes place in vivo, we used chromatin immunoprecipitation (ChIP) [32] from the cells, transiently transfected with CSL and ICN1 genes. Modeling the interaction between CSL/DNA and ICN1 in vivo without interference of other Notch-related factors presented a challenge. We minimized such interference by using the non Notchexpressing transfected host cell line, HEK293T, which is not expected to express Notch-specific factors in stoichiometric amounts to the overexpressed recombinant ICN1 and CSL. Robust overexpression of ICN1 and CSL genes in HEK293T would dwarf any background activity of the endogenous CSL, present in HEK293T [35], but to clear the background even further we used Flag-tagged human CSL expression (Flag-CSL) for anti-Flag rather than anti-CSL ChIP assay.

Anti-Flag ChIP/PCR from Flag-CSL-transfected cells confirmed that the recombinant Flag-CSL protein is bound to Hes1 promoter (Figure 4(a) lane 7). In contrary, anti-ICN1 ChIP/PCR from the cells co-transfected with CSL and ICN1 genes together was negative, disregarding the type of the antibody, used (Figure 4(a) lanes 4-6 as shown). The result suggested that ICN1 was either unbound to CSL/DNA or the immunoprecipitation of ICN1/CSL/DNA complexes by ICN1 epitops was inefficient. We targeted Flag-epitope after "Flag-CSL + Flag-ICN1" co-transfection-and such ChIP/PCR was positive but HES1 promoter was under-represented in "CSL + ICN1"-transfected cells compared to "CSL only"transfected cells (Figure 4(b) upper panel lanes 1 and 2). Serial testing of anti-Flag ChIP/PCR after co-transfections of cells with Flag-CSL plus either ICN1-HA, 6His-ICN1 or untagged ICN1 suggested that the target epitope that helped bring down the HES1 promoter belonged to Flag-CSL and not to ICN1 (Figure 4(b) lanes 1 and 2, all panels). Anti-ICN1 ChIP was negative not only for "CSL+ICN1" transfected cells (as described) but also for "ICN1 only" transfections (Figure 4(b) lane 3, all panels). Prior to each ChIP experiment, the expression of CSL and ICN1 proteins was confirmed by Western blotting (Figure 4(c), for Flag-CSL and ICN1-HA pro- 


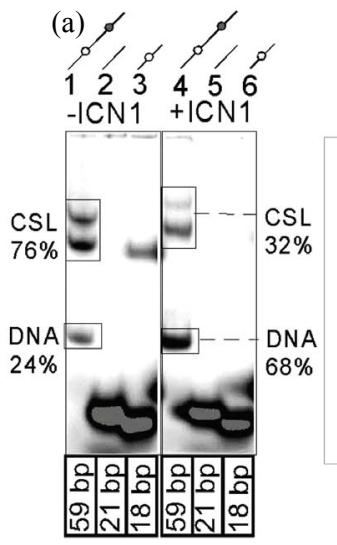

Quantitative to (A) based on 6 experiments:

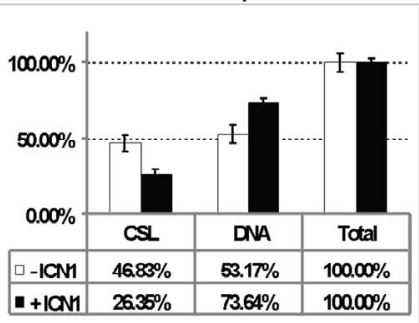

(b)

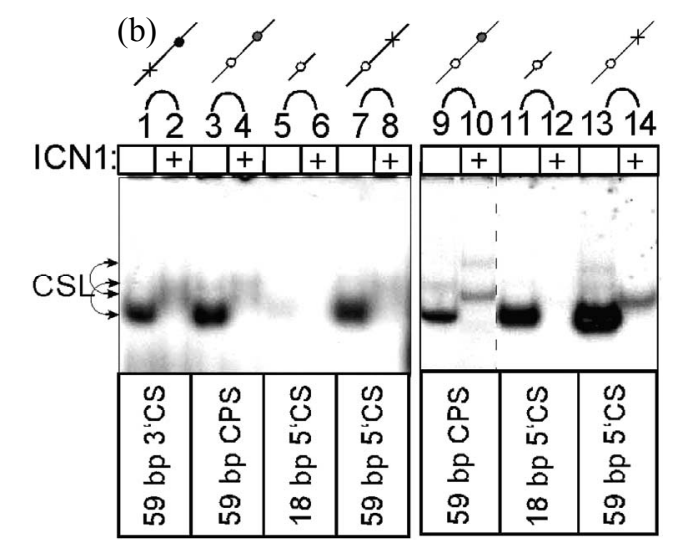

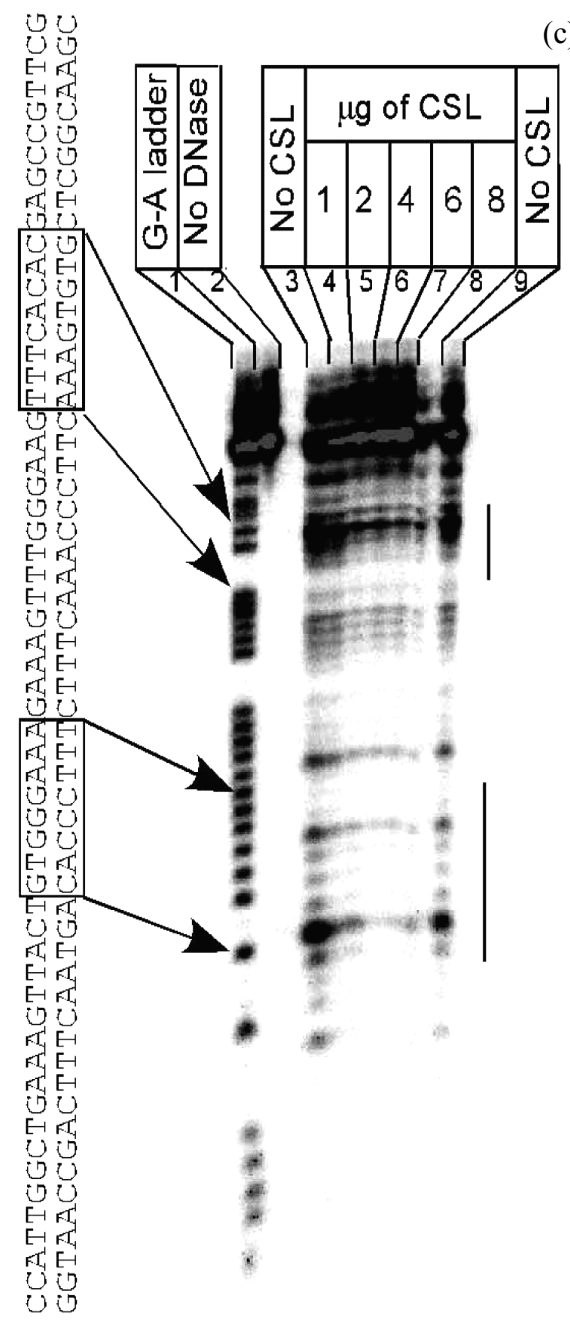

(c)

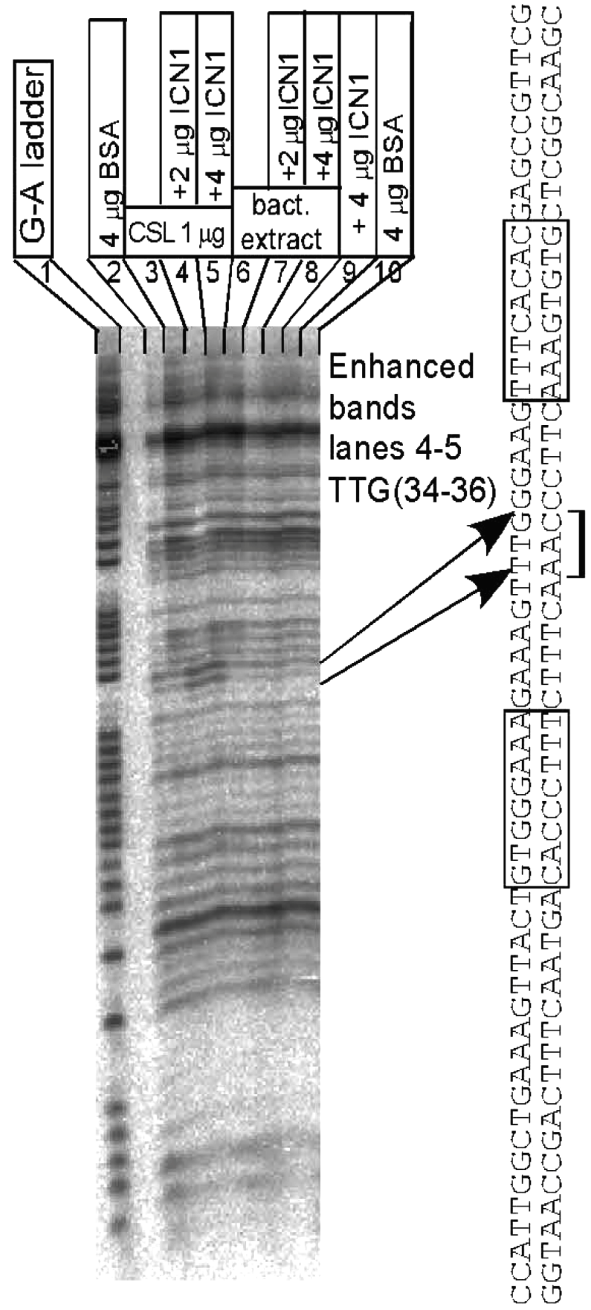

Figure 3. DNA quantification and footprinting analysis. (a) Left panel-EMSA gel of 59 bp CPS probe: lane 1, "DNA/CSL" shift of the "-ICN1" sample contained $76 \%$ of total DNA; lane 4, "DNA/CSL shift of the "+ICN1" sample contained only $32 \%$ of total DNA, indicating dissociation. Right panel: quantification results obtained from six EMSA gels (error bars reflect variations between the samples). Raw data are shown in Table 1 of the Supplement; (b) All 59 bp probes had similar CSL/DNA band shift pattern in presence of ICN1 (CSL/DNA not dissociated completely), different from 18 bp 5 'CS probe (complete CSL/DNA dissociation); (c) Left panel-footprinting analysis with recombinant CSL: the footprints at CSL-binding sites of the 59 bp CPS probe develop increased intensity as CSL concentration rises from 1 to 8 mg (lanes 4 - 8); Right panel footprinting analysis with CSL and ICN1: local disturbance in DNA structure is detected exactly in the middle of CSL paired site, in presence of CSL and ICN1 (lanes 4 and 5). The effect is not observed with ICN1 alone (lane 9), or ICN1 with total protein extract of $E$. coli (lanes 7 and 8), or without ICN1 in presence of CSL (lane 3) or total protein extract of E.coli (lane 6). 


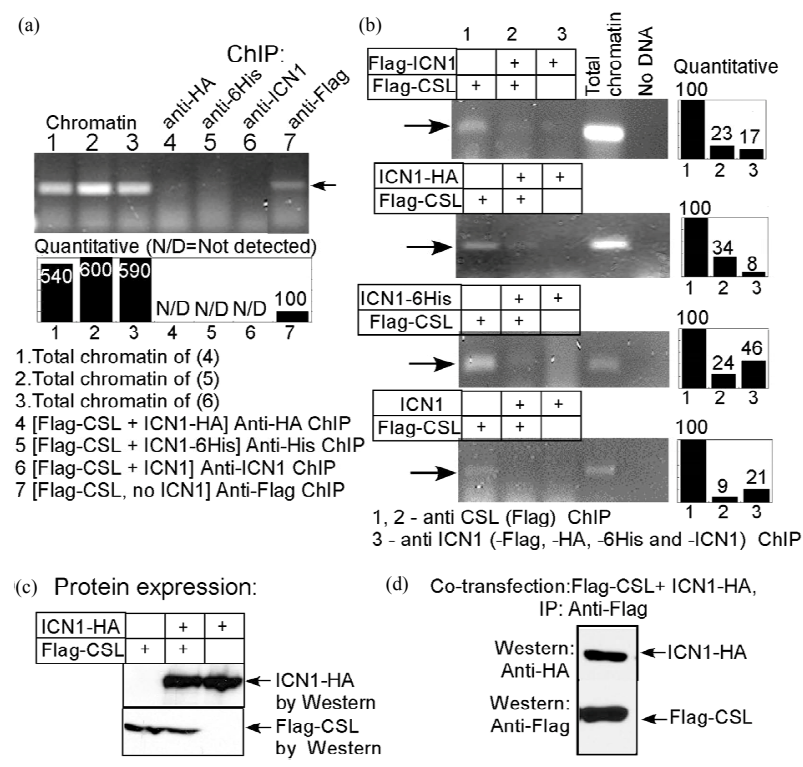

Figure 4. ChIP/PCR of Hes1 promoter. (a) No immunoprecipitation of Hes1 promoter was detected with anti-ICN1 antibodies (lanes 4-6 as marked) from [CSL + ICN1] transfected cells; The immunoprecipitation worked with antiCSL (anti-Flag) antibody from "CSL only" transfected cells; (b) The anti-Flag ChIP/PCR of Hes1 promoter was much more efficient from "Flag-CSL only" transfected cells (lane 1) than from "Flag-CSL + ICN1" co-transfected cells (lane 2) and anti-ICN1 ChIP/PCR was inefficient from "ICN1 only" transfected cells (lane 3). Antibodies and ICN1 species as marked; (c) Expression of the recombinant CSL and ICN1 was confirmed by Western blotting prior to ChIP/ PCR; (d) The recombinant ICN1-HA co-immunoprecipitated from "Flag-CSL + ICN1-HA" transfected cells by anti-Flag IP, confirming accessibility of Flag-epitope of Flag-CSL/ICN1 molecular complex.

teins), excluding the possibility that under-representation of HES1 promoter was caused by the lack of Flag-CSL. Interesting to note that Flag-CSL and ICN1-HA could be co-immunoprecipitated together from the extracts of co-transfected HEK293T, as detected by Western blotting (Figure 4(d)), indicating that Flag-tag epitope of the Flag-CSL was accessible in the Flag-CSL/ICN1-HA protein pair complex, at least when unbound to DNA.

In summary to the in vivo results, we concluded that in the transfected HEK293T cells 1) ICN1 was not associated with HES1 promoter, 2) ICN1 was not associated with CSL/DNA complex at HES1 promoter, and 3) ICN1 caused partial dissociation of CSL from HES1 promoter. To further substantiate these conclusions, we analyzed the transfected HEK293T extracts in EMSA.

\subsection{EMSA with Transfected HEK293T Extracts Is Similar to the One with Purified ICN1, Except Larger Magnitude of Retardation of CSL/DNA Band}

CSL-expressing HEK293T extract (CSL/293T) was al- ready tested in EMSA alongside bacterially expressed purified CSL-6His (i.e., Figure 1(b), etc.). Here again, CSL/293T produced primary EMSA shift (Figure 5(a) lane 1). But neither untransfected cell extract (HEK293T) no cell extract transfected with ICN1 gene only (ICN1/ 293T) had any specific effect on the DNA ${ }^{* *}$ (Figure 5(a)

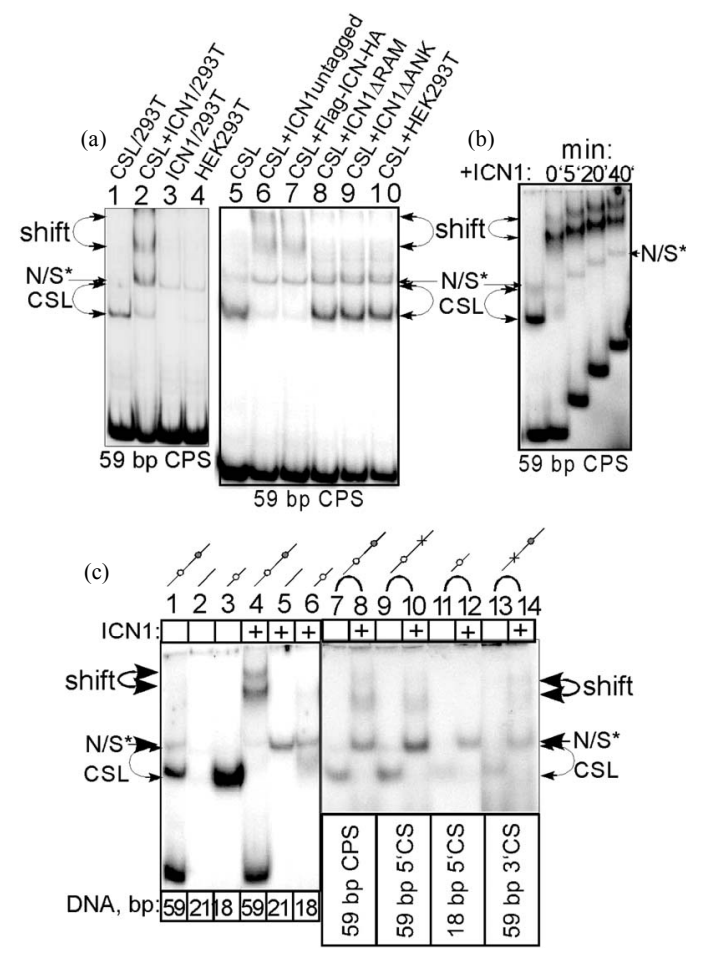

Figure 5. EMSA with HEK293T extracts. (a) "CSL only" transfected HEK293T cell extract produced primary CSL shift in EMSA (lanes 1 and 5) while "ICN1 only" (lane 3) and untransfected HEK293T (lane 4 and 10) extracts produced no specific shifts; Cellular extract of HEK293T cotransfected with "CSL+ICN1" produced primary and secondary EMSA shifts (lane 2); Similar primary and secondary shifts were also observed with CSL/293T extract combined with ICN1/293T extract (lanes 6 and 7); mutant DRAM (lane 8) and DANK (lane 9) ICN1 species caused no secondary shifts of CSL/DNA; (b) Time course: Addition of ICN1/293T extract to pre-incubated CSL/DNA caused secondary EMSA shift when loaded in EMSA gel at " 0 " time and was unchanged in $40 \mathrm{~min}$ (as marked); (c) Testing of different DNA probes with CSL/293T-for primary and CSL/293T + ICN1/293T-for secondary shifts: The 59 bp CPS probe had double-banded primary and double-banded secondary shift (lanes 1 and 4, 7 and 8); 18 bp 5'CS probe had a single-banded primary and no secondary shift (lanes 3 and 6, 11 and 12) and the 59 bp 5'CS and 59 bp 3'CS both had a single-banded primary but double-banded secondary shift (lanes 9, 10 and 13, 14). N/S*-“Non-specific” DNAbinding activity of $293 \mathrm{~T}$ extract, located very closely to 2XCSL/DNA band.

\footnotetext{
${ }^{* *}$ All HEK293T extracts produced a non-specific (N/S) shift in EMSA, running close to $2 \times \mathrm{CSL} / \mathrm{DNA}$ band. This N/S shift was also seen with 7 random DNA probes not containing CSs (in separate testing) and therefore was ignored as a general background.
} 
lanes 3 and 4). When the extract of the cells, co-transfected with CSL+ICN1 genes (CSL+ICN1)/293T (Figure 5(a) lane 2) or the mixture of ICN1/293T + CSL/293T extracts (Figure 5(a) lanes 6 and 7 for tagged and untagged modifications of ICN1) were used in EMSA, CSL/DNA band was supershifted much higher in the gel: the effect resembling that of bacterially expressed/purified ICN1 except the larger magnitude of retardation. RAM- and Ankyrin-deletions of ICN1 (ICN1DRAM/ 293T and ICN1DANK/293T) abolished the effect of WT ICN1/293T (Figure 5(a) lanes 8 and 9), pointing to a direct involvement of ICN1 in the supershifting. Shorter or longer exposure of pre-formed CSL/ $59 \mathrm{bp}$ CPS to WT ICN1/293T had no difference: the full effect took place at "zero time" to the same extent as after 40 minutes of pre-incubation (Figure 5(b)).

We compared the effects of the "59 bp CPS", "21 bp No CS" and " 18 bp 5'CS" probes- and the results were similar to bacterially expressed/purified CSL and ICN1 (except the depth of retardation of the CSL/DNA shift): there was a primary CSL/DNA shift formed with the "59 bp CPS" and " 18 bp 5'CS" probes, and not with the " 21 bp No CS" probe (Figure 5(c) lanes 1-3); two characteristic bands were seen in the shift with the "59 bp CPS" (Figure 5(c) lane 1) and only one band-with the "18 bp CS" (Figure 5(c) lane 3); The addition of ICN1/293T caused retardation of CSL/DNA band in case of CSL/59 bp CPS probe (Figure 5(c) lane 4) and disappearance of CSL/DNA band in case of CSL/18 bp 5'CS band (Figure 5(c) lane 6).

As inferred from ChIP/PCR data (in Section 2.5), ICN1/293T extract induced obvious dissociation of CSL/ DNA molecular complex in case of the $18 \mathrm{bp} 5^{\prime} \mathrm{CS}$ probe, but the super-retardation of the $59 \mathrm{bp}$ CPS without an apparent reduction of DNA material in the shift had to be explained.

We compared the 18 bp 5'CS probe with other CS probes in EMSA with ICN1/293T (Figure 5(c) right panel): Unlike the $18 \mathrm{bp} 5^{\prime} \mathrm{CS}$ probe, the $59 \mathrm{bp} \mathrm{5'CS}$ and 59 bp 3'CS probes did not show obvious signs of dissociation (Figure 5(c) lanes 10 and 14 vs lane 12), implying the involvement of extra sequence of $59 \mathrm{bp}$ probe in maintaining stability during the interaction with ICN1. Surprisingly, both 59 bp CS probes performed identically to the 59 bp CPS probe by producing the double-banded pattern of the super-retarded shift (Figure 5(c) lanes 8, 10 and 14). In EMSA with purified CSL and ICN1 (as described in Section 2.2), the double-banded pattern of the CSL/DNA shift was due to two CSL binding sites present in one probe (the exclusive feature of the CPS probe), but that did not seem to be the case for the double-banded pattern of the super-retarded shift in EMSA with HEK293T extracts. Rather, they represented either $\mathrm{ICN} 1 / \mathrm{CSL} / \mathrm{DNA}+\mathrm{X} / \mathrm{ICN} 1 / \mathrm{CSL} / \mathrm{DNA}$ or X/CSL/DNA +
X/DNA molecular complexes, where "X"-unidentified factor(s) from HEK293T extract. We further elucidated the possibilities using EMSA/Western blotting and DNA quantification.

\subsection{EMSA/Western Blotting Reveals Presence of CSL but Not ICN1 in the Retarded Shift; CSL Is Detected in Only One of the Two Bands of the Shift}

We identified the positions of ICN1-HA and Flag-CSL proteins in EMSA gel using EMSA/Western blotting technique. As seen in the Figure 6(a) (anti-HA Western: lane $1=\mathrm{ICN} 1$ without DNA, lane $2=\mathrm{ICN} 1+\mathrm{DNA}$, lane $3=\mathrm{ICN} 1+\mathrm{CSL} / \mathrm{DNA}$ ), the ICN1-HA protein was not co-localized in the DNA shifts (lane 3), indicating that molecular complexes were unlikely to contain ICN1 (i.e., not ICN1/CSL/DNA or X/ICN1/CSL/DNA, etc.).

Flag-CSL protein, in contrary, was detected in the super-retarded shift of "ICN1/293T sample (Figure 6(a) anti-Flag Western, lane 3; Figure 6(b) anti-Flag Western, lane 3) as well as in the primary shifts (Figure 6(b) anti-Flag Western lane 2). To closer examine the two bands of the shift we resolved them by extended EMSA run in $4 \%$ polyacrylamide gel and subjected to anti-Flag Western analysis (Figure 6(b) lanes 4 and 5): interestingly, Flag-CSL protein was detected only in the upper but not in the lower band (Figure 6(b) lane 5, right panel), consistent with the presumed CSL-heterogeneity of the shift (i.e., CSL/X /DNA and X /DNA molecular complexes, as depicted in the right-side diagram in Figure 6(b)).

\subsection{DNA Quantification Provides Evidence of Partial CSL Dissociation from CSL/X/DNA in Presence of ICN1/293T}

Quantitative analysis revealed higher contents of ${ }^{32} \mathrm{P}$-labeled DNA probe in the super-retarded shift of the "+ICN1/293T" sample than in the primary CSL/DNA shift of "No ICN1" sample $(40 \%$ of the total in lane 2 compared 34\% in Figure 6(c)). Since the amounts of CSL protein were strictly equalized between the samples in lanes 1 and 2, at least some of the DNA in the super-retarded shift $(6 \%$ or more of the total in Figure 6(c)) had to be bound in the protein complexes without CSL: Subsequently, the CSL-heterogeneity of the shift, detected by Western blotting, was supported by quantification of DNA in EMSA bands. Presuming that the lower band of the super-retarded shift is X/DNA (no CSL, no ICN1) molecular complex, and the upper bandCSL/X/DNA molecular complex, the amount of CSL in the DNA-bound molecular complex of " $+\mathrm{ICN} 1 / 293 \mathrm{~T}$ " sample (CSL/X/DNA) can be compared with the amount of CSL in the "No ICN1" sample (CSL/DNA) by quanti- 

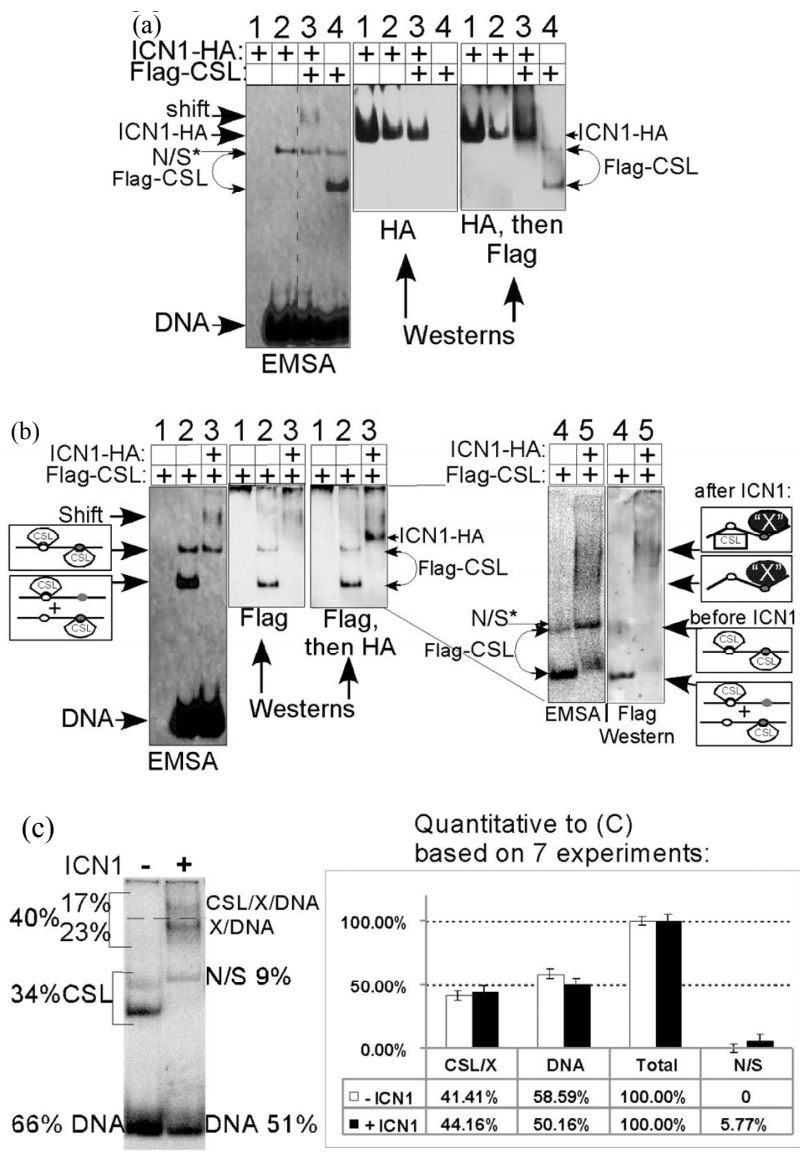

Figure 6. Localization of ICN1 and CSL by Western blotting. (a) Localization of ICN1-HA protein of ICN1-HA/ 293T extract: Left panel- ${ }^{32} P$ exposure of EMSA gel, middle panel-anti-HA Western blotting of the corresponding transfer on the PVDF membrane, right panel-anti-HA and then anti-Flag Western of the same membrane. Lanes: 1, ICN1HA/293T without DNA; 2, ICN1-HA/293T + DNA; 3, ICN1HA/293T + CSL/293T/DNA; 4, CSL/293T/DNA; (b) Localization of Flag-CSL protein of Flag-CSL/293T extract: Panel (from the left)-EMSA gel; panel 2, anti-Flag Western blotting; panel 3, anti-Flag then anti-HA Western of the same protein transfer; panel 4, extended EMSA run; panel 5, anti-Flag Western of the extended EMSA. Lanes: 1, FlagCSL/293T without DNA; 2, Flag-CSL/293T+DNA; 3, FlagCSL/293T+DNA + ICN1-HA/293T; 4, Flag-CSL/293T + DNA (extended EMSA run); 5, Flag-CSL/293T + DNA + ICN1-HA/293T (extended EMSA run); (c) Quantification of ${ }^{32} \mathrm{P}$-activity in EMSA bands, produced by HEK293T extracts. In the left panel-a selected picture of EMSA gel used in quantification: Lanes: 1, CSL/293T + DNA with no ICN1; 2, CSL/293T+DNA + ICN1/293T. In the right panel-combined results of seven independent experiments in one plot with error bars representing variability between the samples. N/S - “Non-specific" DNA-binding activity of $293 \mathrm{~T}$ extract.

fication the equamolar amounts of the DNA in the correspondent EMSA bands. Interestingly, with such presumption only $\sim$ half of the CSL is still bound to the DNA in the "+ICN1/293T" sample compared to "No ICN1" sample (17\% of CSL/X/DNA in lane 2 vs $34 \%$ of CSL/ DNA in lane 1, Figure 6(c)), meaning that CSL dissociated in presence of ICN1: consistent with ChIP/PCR data and remarkably similar to the EMSA results obtained with purified ICN1 and CSL.

\section{Discussion}

The goal of this study was to evaluate the impact of fulllength human ICN1 protein on CSL/DNA molecular complex. The experimental results reveal that ICN1 may function alone to allosterically modulate CSL/DNA complex, at HES1 promoter. Specifically, the data here suggest that full length human ICN1 does not form a stable association with CSL/DNA but induces a conformational change in CSL/DNA; the ICN1-induced CSLDNA conformation is unstable and prone to dissociation; the bond between the CSL and DNA in the new conformation may involve HES1 promoter sites other than the consensus CSL-binding sites. Transient dissociation of the CSL/DNA complex may sponsor the recruitment of additional factors and DNA sequences.

We acknowledge that several research groups have reported detectable ICN/CSL/DNA shifts in EMSA [20, 29], although the lack of binding of ICN1 to DNA/CSL was also reported: [22]. A number of reasons may account for these differences, amongst them:

- Human ICN1 and non-human ICN1 may possess different binding affinities/stoichiometry for the CSL/ DNA;

- In the most studies, the ICN1/CSL/DNA molecular complex was reportedly assembled with truncated ICN1 (ANK, RAM or RAMANK domains, etc.): The use of a non-truncated, full-length human ICN1 could alter protein-protein interaction and binding with CSL/DNA;

- The in vitro setup and DNA probes are not identical and often differ between laboratories and this can lead to discrepancies in data outcomes;

- A conformational change in CSL/DNA could decrease electrophoretic mobility, and this may be falsely identified as the ICN1/CSL/DNA molecular complex in EMSA: the presence of ICN1 in the shift must be verified;

An excellent illustration of the interspecies differences is provided in the recent publication by Kovall and coworkers [36] wherein they report a 50-fold difference in affinity of the RAM domain to CSL between the worm and mouse species. In the same study, the reported ability of RAM domain to induce modulation of CSL/DNA is only $50 \%$ compared to the extent of modulation found in the ternary complex (MAML/ICN/CSL + DNA) with RAMANK-can serve as an illustration of change that is initially influenced by the size of the truncated ICN1. Of course, in the latter case there is also unknown contribu- 
tion of MAML protein.

The binding of MAML to CSL/DNA is dependent upon the participation of ICN1, because it requires opening the loop in N-terminal domain of CSL that remains closed in the free CSL/DNA form, as presumed from crystallization studies [30,37]. The instability of CSL/DNA, detected in our experiments, implies that CSL conformation in the MAML/ICN1/CSL/DNA complex (as seen in crystal structure) is different from the conformation induced by ICN1 in the absence MAML, because the ternary complex is not characterized by low affinity to DNA. Instead, CSL prints in DNA footprinting are enhanced with MAML [20]. Thus, it is likely that MAML, in turn, re-configures the CSL/DNA complex that is modulated by ICN1, and stabilizes the bond of CSL with DNA. Our model presumes that highest level of cooperation between ICN1 and MAML is required to produce maximal affinity for binding to CSL/DNA. The interaction of ICN1 with DNA/CSL lasts seconds, taking into account that the mixed (ICN1 + DNA/CSL) samples were applied immediately to the EMSA [the interacting molecules are "fixed" together by "caging effect" after entering the gel [38] and the time of entry into the gel lasted less than 1 minute. ICN1 opens CSL conformation and compromises the CSL bond with the promoter DNA. The allosteric modulation of CSL/DNA that is induced in the presence of ICN1 may prove to be of higher significance than physical association of ICN1 with the CSL/ DNA molecular complex. Jones and co-workers have discussed that "transactivation" by Notch is achieved through recruitment of available non-DNA-binding co activators such as MAML, SKIP, Deltex, and not by functionality of ICNs own domains [20]. There is a growing family of factors, known to interact with Notch, whose function is not completely understood. MAML proteins interact with partners other than Notch and CSL, and the Notch pathway that competes for MAML1, titrates this protein molecule from interacting with the other transcription factors [39]. SKIP, binding to CSL and ICN, may well prove to be another "stabilizer" of ICN1-CSL/DNA, alternative to MAML, but crystal structure of SKIP/ICN1/ CSL/DNA is yet to be determined. Deltex does not bind to CSL [40], but may act with ICN to modulate other enhancer-binding proteins. Many genes that contain CSLbinding sites in their promoters are not always responsive to ICN, despite the presence of CSL in the nuclei of the cells $[41,42]$; The ability of Notch to modify CSL/DNA, and potentially, "Another Effector/DNA", opens an intriguing possibility that activation of Notch-regulated promoters is achieved through recruitment of multiple different, promoter-specific co-activators (MAML, SKIP, Deltex, etc.), which may take advantage of the universal function of ICN1 as "modulator" of enhancer-binding protein/DNA complexes (including the CSL/DNA). In some cases, "opening" of an enhancer-binding protein (such as CSL) may be the only necessary and sufficient role of ICN1; In the case of HES1 transcription, ICN1 actively modulates CSL into a configuration that accepts the transcription co-activator MAML, plus ICN1: if MAML is available and appropriately positioned at the time of ICN1-CSL/DNA interaction, the "open" conformation is re-adjusted and a stable MAML/ICN1/CSL/ DNA transcription complex is formed; if MAML is unavailable - CSL may dissociate or revert to the "free DNAbound" conformation, and thereby inhibit transcription. Recent studies reveal that the consensus CSL binding sequences are present in hundreds if not thousands promoters of different genes, and CSL occupancy of those promoters is quite high $[43,44]$; Thus, the differential expression of promoter-specific CSL/DNA-stabilizing factors may even form the basis of precise response(s) to Notch signaling.

The Notch-induced instability/modulation of CSL/ DNA promoter complexes as demonstrated in this study contributes to our understanding of how Notch, when expressed in small amounts within the nucleus, can displace CSL-bound co-repressor proteins that exist at much higher concentrations [2]. The current theory is that SKIP protein assists Notch in the displacement of co-repressors from CSL [45]. Another possibility is that allosteric modulation of CSL/DNA can render a conformation not favorable to binding of co-repressor complexes, thus causing their dissociation. Or even that the CSL may dissociate from the promoter itself upon ICN1 impact, vacating the place to pre-assembled MAML/ICN1/CSL molecular complexes, whose existence is in discussion in the alternative model of Notch activation [36,46,47], different from the model where CSL is constantly statically bound to DNA.

\section{Acknowledgements}

This work was supported by NCI (NIH) grant 5 R01 CA 06245011.

\section{REFERENCES}

[1] G. Weinmaster and C. Kintner, "Modulation of Notch Signaling during Somitogenesis," Annual Review of Cell and Developmental Biology, Vol. 19, 2003, pp. 367-395. doi:10.1146/annurev.cellbio.19.111301.115434

[2] J. S. Mumm and R. Kopan, "Notch Signaling: From the Outside in," Developmental Biology, Vol. 228, No. 2, 2000, pp. 151-165. doi:10.1006/dbio.2000.9960

[3] M. V. Gustafsson, et al., "Hypoxia Requires Notch Signaling to Maintain the Undifferentiated Cell State," Developmental Cell, Vol. 9, No. 5, 2005, pp. 617-628. doi:10.1016/j.devcel.2005.09.010

[4] G. D. Hurlbut, et al., "Crossing paths with Notch in the 
Hyper-Network," Current Opinion in Cell Biology, Vol. 19, No. 2, 2007, pp. 166-175. doi:10.1016/j.ceb.2007.02.012

[5] F. Jundt, R. Schwarzer and B. Dorken, "Notch Signaling in Leukemias and Lymphomas," Current Molecular Medicine, Vol. 8, No. 1, 2008, pp. 51-59. doi:10.2174/156652408783565540

[6] R. M. Demarest, F. Ratti and A. J. Capobianco, "It's TALL about Notch," Oncogene, Vol. 27, No. 38, 2008, pp. 5082-5091. doi:10.1038/onc.2008.222

[7] F. Wu, A. Stutzman and Y. Y. Mo, "Notch Signaling and Its Role in Breast Cancer," Frontiers in Bioscience, Vol. 12, 2007, pp. 4370-4383. doi:10.2741/2394

[8] M. Roy, W. S. Pear and J. C. Aster, "The Multifaceted Role of Notch in Cancer," Current Opinion in Genetics \& Development, Vol. 17, No. 1, 2007, pp. 52-59. doi:10.1016/i.gde.2006.12.001

[9] U. Koch and F. Radtke, "Notch and Cancer: A DoubleEdged Sword," Cellular and Molecular Life Sciences, Vol. 64, No. 21, 2007. pp. 2746-2762. doi:10.1007/s00018-007-7164-1

[10] Z. Wang, et al., "Exploitation of the Notch Signaling Pathway as a Novel Target for Cancer Therapy," Anticancer Research, Vol. 28, No. 6A, 2008, pp. 3621-3630.

[11] M. A. Villaronga, C. L. Bevan and B. Belandia, "Notch Signaling: A Potential Therapeutic Target in Prostate Cancer," Current Cancer Drug Targets, Vol. 8, No. 7, 2008, pp. 566-580. doi:10.2174/156800908786241096

[12] P. Rizzo, et al., "Rational Targeting of Notch Signaling in Cancer," Oncogene, Vol. 27, No. 38, 2008, pp. 51245131. doi:10.1038/onc.2008.226

[13] R. Kopan and M. X. Ilagan, "The Canonical Notch Signaling Pathway: Unfolding the Activation Mechanism," Cell, Vol. 137, No. 2, 2009, pp. 216-233. doi:10.1016/j.cell.2009.03.045

[14] M. E. Fortini, "Notch Signaling: The Core Pathway and Its Posttranslational Regulation," Developmental Cell, Vol. 16, No. 5, 2009, pp. 633-647. doi:10.1016/j.devcel.2009.03.010

[15] T. Borggrefe and F. Oswald, "The Notch Signaling Pathway: Transcriptional Regulation at Notch Target Genes," Cellular and Molecular Life Sciences, Vol. 66, No. 10, 2009, pp. 1631-1646. doi:10.1007/s00018-009-8668-7

[16] M. Le Gall and E. Giniger, "Identification of Two Binding Regions for the Suppressor of Hairless Protein within the Intracellular Domain of Drosophila Notch," The Journal of Biological Chemistry, Vol. 279, No. 28, 2004, pp. 29418-29426. doi:10.1074/jbc.M404589200

[17] H. Y. Kao, et al., "A Histone Deacetylase Corepressor Complex Regulates the Notch Signal Transduction Pathway," Genes \& Development, Vol. 12, No. 15, 1998, pp. 2269-2277. doi:10.1101/gad.12.15.2269

[18] J. J. Hsieh, et al., "CIR, a Corepressor Linking the DNA Binding Factor CBF1 to the Histone Deacetylase Complex," Proceedings of the National Academy of Sciences of USA, Vol. 96, No. 1, 1999, pp. 23-28.

doi:10.1073/pnas.96.1.23
[19] F. Oswald, et al., "RBP-Jkappa/SHARP Recruits CtIP/ CtBP Corepressors to Silence Notch Target Genes," Molecular and Cellular Biology, Vol. 25, No. 23, 2005, pp. 10379-10390.

doi:10.1128/MCB.25.23.10379-10390.2005

[20] C. J. Fryer, et al., "Mastermind Mediates ChromatinSpecific Transcription and Turnover of the Notch Enhancer Complex," Genes \& Development, Vol. 16, No. 11, 2002, pp. 1397-1411. doi:10.1101/gad.991602

[21] A. G. Petcherski and J. Kimble, "Mastermind Is a Putative Activator for Notch," Current Biology, Vol. 10, No. 13, 2000, pp. R471-473. doi:10.1016/S0960-9822(00)00577-7

[22] L. Wu, et al., "MAML1, a Human Homologue of Drosophila Mastermind, Is a Transcriptional Co-Activator for NOTCH Receptors," Nature Genet, Vol. 26, No. 4, 2000, pp. 484-489. doi:10.1038/82644

[23] S. Zhou, et al., "A Role for SKIP in EBNA2 Activation of CBF1-Repressed Promoters," Journal of Virology, Vol. 74, No. 4, 2000, pp. 1939-1947. doi:10.1128/JVI.74.4.1939-1947.2000

[24] L. Espinosa, et al., "Phosphorylation by Glycogen Synthase Kinase-3 Beta Down-Regulates Notch Activity, a Link for Notch and Wnt Pathways," Journal of Biological Chemistry, Vol. 278, No. 34, 2003, pp. 32227-32235. doi:10.1074/jbc.M304001200

[25] H. Qin, et al., "RING1 Inhibits Transactivation of RBP-J by Notch through Interaction with LIM Protein KyoT2," Nucleic Acids Research, Vol. 32, No. 4, 2004, pp. 14921501. doi:10.1093/nar/gkh295

[26] F. Oswald, et al., "p300 Acts as a Transcriptional Coactivator for Mammalian Notch-1," Molecular and Cellular Biology, Vol. 21, No. 22, 2001, pp. 7761-7774. doi:10.1128/MCB.21.22.7761-7774.2001

[27] E. A. Johnson, "HIF Takes It Up a Notch," Science Signal, Vol. 4, No. 181, 2011, p. pe33. doi: $10.1126 /$ scisignal.2002277

[28] C. J. Fryer, J. B. White and K. A. Jones, Mastermind Recruits CycC:CDK8 to Phosphorylate the Notch ICD and Coordinate Activation with Turnover," Molecular Cell, Vol. 16, No. 4, 2004, pp. 509-520.

doi:10.1016/j.molcel.2004.10.014

[29] Y. Nam, et al., "Structural Basis for Cooperativity in Recruitment of MAML Coactivators to Notch Transcription Complexes," Cell, Vol. 124, No. 5, 2006, pp. 973983. doi:10.1016/j.cell.2005.12.037

[30] R. A. Kovall and W. A. Hendrickson, "Crystal Structure of the Nuclear Effector of Notch Signaling, CSL, Bound to DNA," The EMBO Journal, Vol. 23, No. 17, 2004, pp. 3441-3451. doi:10.1038/sj.emboj.7600349

[31] J. J. Wilson and R. A. Kovall, "Crystal Structure of the CSL-Notch-Mastermind Ternary Complex Bound to DNA," Cell, Vol. 124, No. 5, 2006, pp. 985-996. doi:10.1016/j.cell.2006.01.035

[32] A. S. Weinmann, et al., "Use of Chromatin Immunoprecipitation to Clone Novel E2F Target Promoters," Molecular and Cellular Biology, Vol. 21, No. 20, 2001, pp. 6820-6832. doi:10.1128/MCB.21.20.6820-6832.2001 
[33] D. T. Nellesen, E.C. Lai and J.W. Posakony, "Discrete Enhancer Elements Mediate Selective Responsiveness of Enhancer of Split Complex Genes to Common Transcriptional Activators," Developmental Biology, Vol. 213, No. 1, 1999, pp. 33-53. doi:10.1006/dbio.1999.9324

[34] A. M. Bailey and J. W. Posakony, "Suppressor of Hairless Directly Activates Transcription of Enhancer of Split Complex Genes in Response to Notch Receptor Activity," Genes and Development, Vol. 9, No. 21, 1995, pp. 2609-2622. doi:10.1101/gad.9.21.2609

[35] S. Jeffries, D. J. Robbins and A. J. Capobianco, "Characterization of a High-Molecular-Weight Notch Complex in the Nucleus of Notch(ic)-Transformed RKE Cells and in a Human T-Cell Leukemia Cell Line," Molecular and Cellular Biology, Vol. 22, No. 11, 2002, pp. 3927-3241. doi:10.1128/MCB.22.11.3927-3941.2002

[36] D. R. Friedmann, J. J. Wilson and R. A. Kovall, "RAMInduced Allostery Facilitates Assembly of a Notch Pathway Active Transcription Complex," The Journal of Biological Chemistry, Vol. 283, No. 21, 2008, pp. 1478114791. doi:10.1074/jbc.M709501200

[37] R. A. Kovall, "Structures of CSL, Notch and Mastermind Proteins: Piecing Together an Active Transcription Complex," Current Opinion in Structural Biology, Vol. 17, No. 1, 2007, pp. 117-127. doi:10.1016/j.sbi.2006.11.004

[38] M. Fried and D. M. Crothers, "Equilibria and Kinetics of Lac Repressor-Operator Interactions by Polyacrylamide Gel Electrophoresis," Nucleic Acids Research, Vol. 9, No. 23, 1981, pp. 6505-6525. doi:10.1093/nar/9.23.6505

[39] A. S. McElhinny, J. L. Li and L. Wu, "Mastermind-Like Transcriptional Co-Activators: Emerging Roles in Regulating Cross Talk among Multiple Signaling Pathways," Oncogene, Vol. 27, No. 38, 2008, pp. 5138-5147. doi:10.1038/onc.2008.228

[40] N. Yamamoto, et al., "Role of Deltex-1 as a Transcrip- tional Regulator Downstream of the Notch Receptor," The Journal of Biological Chemistry, Vol. 276, No. 48, 2001, pp. 45031-45040. doi:10.1074/jbc.M105245200

[41] J. W. Cave, et al., "A DNA Transcription Code for Cell-Specific Gene Activation by Notch Signaling," Current Biology, Vol. 15, No. 2, 2005, pp. 94-104. doi:10.1016/j.cub.2004.12.070

[42] A. Neves and J. R. Priess, "The REF-1 Family of bHLH Transcription Factors Pattern C. elegans Embryos through Notch-Dependent and Notch-Independent Pathways," Developmental Cell, Vol. 8, No. 6, 2005, pp. 867-879. doi:10.1016/j.devcel.2005.03.012

[43] L. M. Persson and A. C. Wilson, "Wide-Scale Use of Notch Signaling Factor CSL/RBP-Jkappa in RTA-Mediated Activation of Kaposi's Sarcoma-Associated Herpesvirus Lytic Genes," Journal of Virology, Vol. 84, No. 3, 2009, pp. 1334-1347. doi:10.1128/JVI.01301-09

[44] H. Hamidi, et al., "Identification of Novel Targets of CSL-Dependent Notch Signaling in Hematopoiesis," PLoS ONE, Vol. 6, No. 5, 2011, p. e20022. doi:10.1371/journal.pone.0020022

[45] S. Zhou, et al., "SKIP, a CBF1-Associated Protein, Interacts with the Ankyrin Repeat Domain of NotchIC to Facilitate NotchIC Function," Molecular and Cellular Biology, Vol. 20, No. 7, 2000, pp. 2400-2410. doi:10.1128/MCB.20.7.2400-2410.2000

[46] A. Krejci and S. Bray, "Notch Activation Stimulates Transient and Selective Binding of $\mathrm{Su}(\mathrm{H}) / \mathrm{CSL}$ to Target Enhancers," Genes \& Development, Vol. 21, No. 11, 2007, pp. 1322-1327. doi:10.1101/gad.424607

[47] Y. Nam, et al., "Cooperative Assembly of Higher-Order Notch Complexes Functions as a Switch to Induce Transcription," Proceedings of the National Academy of Sciences of the United States of America, Vol. 104, No. 7, 2007, pp. 2103-2108. doi:10.1073/pnas.0611092104 


\section{Supplement}

1) ICN1 and CSL recombinant gene sequences used for expression in HEK293T cells and E. coli strain BL21DE3. $\mathrm{N}$-terminal ICN1 sequence (Flag- epitope) added when cloned in pcDNA3.1:

HindIII BamHI

AAGCTTCCACCATGGATTACAAGGATGACGACGATAAGGGATCC

$$
\begin{array}{lllllllllll}
M & D & Y & K & D & D & D & D & K & G & S
\end{array}
$$

N-terminal ICN1 sequence (with 6xHis epitope) added when cloned in pET28a:
$3 / 1$ $33 / 11$ $63 / 21$
$93 / 31$ BamHI
atg gct agc atg act ggt gga cag caa atg ggt cgc gga tcc
met ala ser met thr gly gly gln gln met gly arg gly ser

atg gge agc agc cat cat cat cat cat cac agc agc gge ctg gtg ccg cgc gge agc cat Met gly ser ser his his his his his his ser ser gly leu val pro arg gly ser his

Sequence Range: 1 to 2482

$$
10 \quad 20 \quad 30 \quad 50
$$

GGATCCGGAGGCATGGGCTCCCGCAAGCGCCGGCGGCAGCATGGCCAGCT CCTAGGCCTCCGTACCCGAGGGCGTTCGCGGCCGCCGTCGTACCGGTCGA

$\begin{array}{llllllllllllllllllll} & G & S & G & G & M & G & S & R & K & R & R & R & Q & H & G & Q & L>\end{array}$ TRANSLATION OF ICN1 [A]

$$
\begin{array}{llll}
60 & 80 & 90 & 100
\end{array}
$$

CTGGTTCCCTGAGGGCTTCAAAGTGTCTGAGGCCAGCAAGAAGAAGCGGC GACCAAGGGACTCCCGAAGTTTCACAGACTCCGGTCGTTCTTCTTCGCCG

$\begin{array}{llllllllllllllll}W & F & P & E & G & F & K & V & S & E & A & S & K & K & K & R>\end{array}$ TRANSLATION OF ICN1 [A]
110
120
130
140
150

GGGAGCCCCTCGGCGAGGACTCCGTGGGCCTCAAGCCCCTGAAGAACGCT CCCTCGGGGAGCCGCTCCTGAGGCACCCGGAGTTCGGGGACTTCTTGCGA $\begin{array}{llllllllllllllllll}R & E & P & L & G & E & D & S & V & G & L & K & P & L & K & N & A>\end{array}$ TRANSLATION OF ICN1 [A]

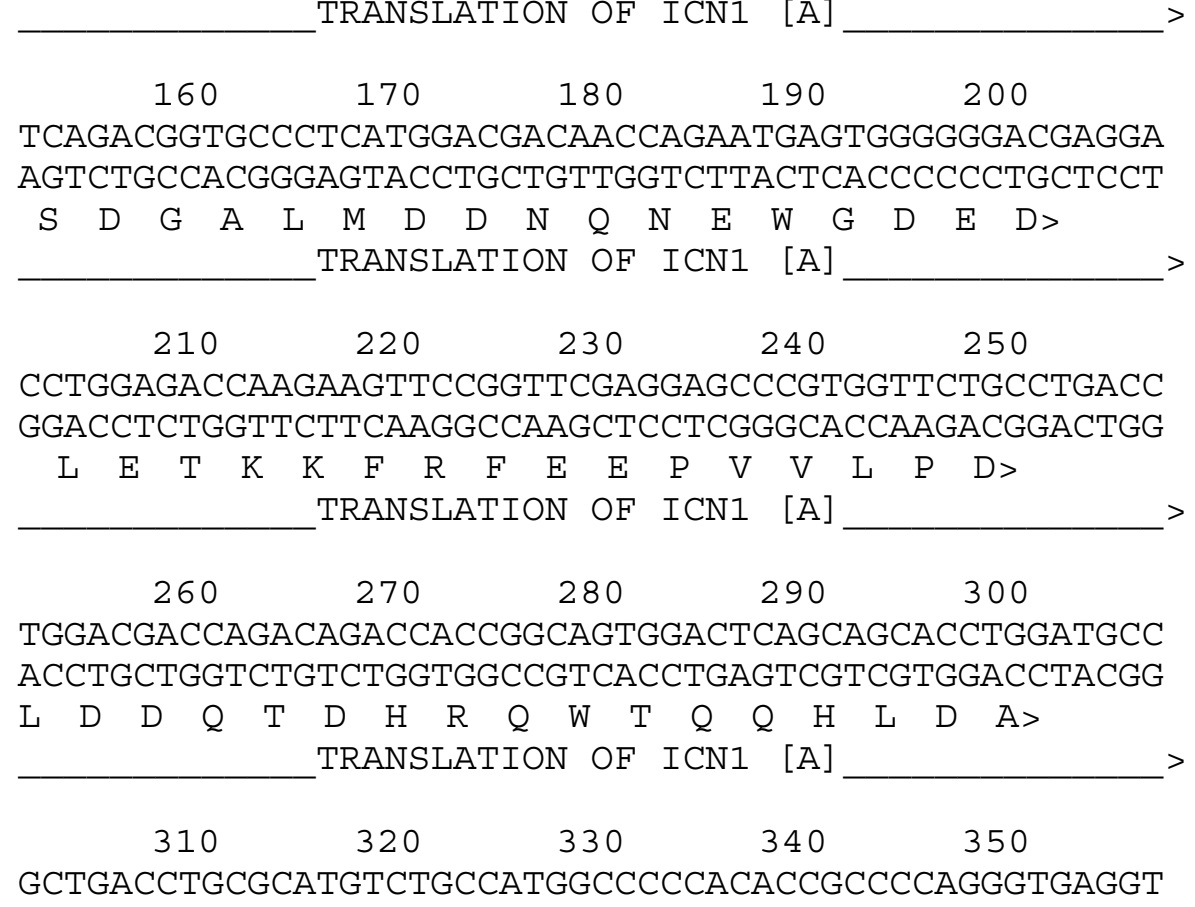


CGACTGGACGCGTACAGACGGTACCGGGGGTGTGGCGGGGTCCCACTCCA $\begin{array}{llllllllllllllllll}A & D & L & R & M & S & A & M & A & P & T & P & P & Q & G & E & V>\end{array}$ TRANSLATION OF ICN1 [A]

$\begin{array}{llll}360 & 370 & 380 & 390\end{array} 400$

TGACGCCGACTGCATGGACGTCAATGTCCGCGGGCCTGATGGCTTCACCC ACTGCGGCTGACGTACCTGCAGTTACAGGCGCCCGGACTACCGAAGTGGG

$\begin{array}{llllllllllllllll}D & A & D & C & M & D & V & N & V & R & G & P & D & G & F & T>\end{array}$ TRANSLATION OF ICN1 [A]
410
420
430
440
450

CGCTCATGATCGCCTCCTGCAGCGGGGGCGGCCTGGAGACGGGCAACAGC GCGAGTACTAGCGGAGGACGTCGCCCCCGCCGGACCTCTGCCCGTTGTCG $\begin{array}{lllllllllllllllll}P & L & M & I & A & S & C & S & G & G & G & L & E & T & G & N & S>\end{array}$ TRANSLATION OF ICN1 [A]

$460 \quad 470 \quad 480 \quad 490 \quad 500$

GAGGAAGAGGAGGACGCGCCGGCCGTCATCTCCGACTTCATCTACCAGGG CTCCTTCTCCTCCTGCGCGGCCGGCAGTAGAGGCTGAAGTAGATGGTCCC

$\begin{array}{llllllllllllllllll}E & E & E & E & D & A & P & A & V & I & S & D & F & I & Y & Q & G>\end{array}$ TRANSLATION OF ICN1 [A]

$510 \quad 520 \quad 530 \quad 540 \quad 550$

CGCCAGCCTGCACAACCAGACAGACCGCACGGGCGAGACCGCCTTGCACC GCGGTCGGACGTGTTGGTCTGTCTGGCGTGCCCGCTCTGGCGGAACGTGG

$\begin{array}{llllllllllllllll}A & S & L & H & N & Q & T & D & R & T & G & E & T & A & L & H>\end{array}$ TRANSLATION OF ICN1 [A]

$560 \quad 570 \quad 580 \quad 590 \quad 600$

TGGCCGCCCGCTACTCACGCTCTGATGCCGCCAAGCGCCTGCTGGAGGCC ACCGGCGGGCGATGAGTGCGAGACTACGGCGGTTCGCGGACGACCTCCGG $\begin{array}{lllllllllllllllll}L & A & A & R & Y & S & R & S & D & A & A & K & R & L & L & E & A>\end{array}$ TRANSLATION OF ICN1 [A]
610
620
630
640
650

AGCGCAGATGCCAACATCCAGGACAACATGGGCCGCACCCCGCTGCATGC TCGCGTCTACGGTTGTAGGTCCTGTTGTACCCGGCGTGGGGCGACGTACG $\begin{array}{lllllllllllllllll}S & A & D & A & N & I & Q & D & N & M & G & R & T & P & L & H & A>\end{array}$ TRANSLATION OF ICN1 [A]

$660 \quad 670 \quad 680 \quad 690 \quad 700$

GGCTGTGTCTGCCGACGCACAAGGTGTCTTCCAGATCCTGATCCGGAACC CCGACACAGACGGCTGCGTGTTCCACAGAAGGTCTAGGACTAGGCCTTGG

$\begin{array}{llllllllllllllll}A & V & S & A & D & A & Q & G & V & F & Q & I & L & I & R & N>\end{array}$ TRANSLATION OF ICN1 [A]
710
720
730
740
750

GAGCCACAGACCTGGATGCCCGCATGCATGATGGCACGACGCCACTGATC CTCGGTGTCTGGACCTACGGGCGTACGTACTACCGTGCTGCGGTGACTAG $\begin{array}{lllllllllllllllll}R & A & T & D & L & D & A & R & M & H & D & G & T & T & P & L & I>\end{array}$ TRANSLATION OF ICN1 [A]

$\begin{array}{ccccc}760 & 770 & 780 & 790 & 800 \\ \text { CTGGCTGCCCGCCTGGCCGTGGAGGGCATGCTGGAGGACCTCATCAACTC }\end{array}$


GACCGACGGGCGGACCGGCACCTCCCGTACGACCTCCTGGAGTAGTTGAG

$\begin{array}{lllllllllllllllll}L & A & A & R & L & A & V & E & G & M & L & E & D & L & I & N & S>\end{array}$ TRANSLATION OF ICN1 [A]

$810 \quad 820 \quad 830 \quad 840 \quad 850$

ACACGCCGACGTCAACGCCGTAGATGACCTGGGCAAGTCCGCCCTGCACT TGTGCGGCTGCAGTTGCGGCATCTACTGGACCCGTTCAGGCGGGACGTGA

$\begin{array}{llllllllllllllll}H & A & D & V & N & A & V & D & D & \text { L } & G & K & S & A & L & H>\end{array}$ TRANSLATION OF ICN1 [A]
860
870
880
890
900

GGGCCGCCGCCGTGAACAATGTGGATGCCGCAGTTGTGCTCCTGAAGAAC CCCGGCGGCGGCACTTGTTACACCTACGGCGTCAACACGAGGACTTCTTG $\begin{array}{llllllllllllllllll}\text { W } & A & A & A & V & N & N & V & D & A & A & V & V & L & L & K & N>\end{array}$ TRANSLATION OF ICN1 [A]
910
920
930
940
950

GGGGCTAACAAAGATATGCAGAACAACAGGGAGGAGACACCCCTGTTTCT CCCCGATTGTTTCTATACGTCTTGTTGTCCCTCCTCTGTGGGGACAAAGA $\begin{array}{lllllllllllllllll}G & A & N & K & D & M & Q & N & N & R & E & E & T & P & L & F & L>\end{array}$ TRANSLATION OF ICN1 [A]

$960 \quad 970 \quad 980 \quad 990 \quad 1000$

GGCCGCCCGGGAGGGCAGCTACGAGACCGCCAAGGTGCTGCTGGACCACT CCGGCGGGCCCTCCCGTCGATGCTCTGGCGGTTCCACGACGACCTGGTGA

$\begin{array}{llllllllllllllll}A & A & R & E & G & S & Y & E & T & A & K & V & L & L & D & H>\end{array}$ TRANSLATION OF ICN1 [A]
1010
1020
1030
1040
1050

TTGCCAACCGGGACATCACGGATCATATGGACCGCCTGCCGCGCGACATC AACGGTTGGCCCTGTAGTGCCTAGTATACCTGGCGGACGGCGCGCTGTAG $\begin{array}{lllllllllllllllll}F & A & N & R & D & I & T & D & H & M & D & R & L & P & R & D & I>\end{array}$ TRANSLATION OF ICN1 [A]
1060
1070
1080
1090
1100

GCACAGGAGCGCATGCATCACGACATCGTGAGGCTGCTGGACGAGTACAA CGTGTCCTCGCGTACGTAGTGCTGTAGCACTCCGACGACCTGCTCATGTT
$\begin{array}{llllll}A & Q & E & R & M\end{array}$

$\begin{array}{cccccccc}\text { H } & \text { H } & \text { D } & \text { I } & V & R & L & L \\ \text { TRANSLATION } & \text { OF } & \text { ICN1 } & {[A}\end{array}$

1110

1120

1130

1140

1150

CCTGGTGCGCAGCCCGCAGCTGCACGGAGCCCCGCTGGGGGGCACGCCCA GGACCACGCGTCGGGCGTCGACGTGCCTCGGGGCGACCCCCCGTGCGGGT

$\begin{array}{llllllllllllllll}L & V & R & S & P & Q & \text { L } & \text { H } & \text { G } & \text { A } & \text { P } & \text { L } & G & G & \text { T } & P>\end{array}$ TRANSLATION OF ICN1 [A]
1160
1170
1180
1190
1200

CCCTGTCGCCCCCGCTCTGCTCGCCCAACGGCTACCTGGGCAGCCTCAAG GGGACAGCGGGGGCGAGACGAGCGGGTTGCCGATGGACCCGTCGGAGTTC $\begin{array}{lllllllllllllllll}T & L & S & P & P & L & C & S & P & N & G & Y & L & G & S & L & K>\end{array}$ TRANSLATION OF ICN1 [A]
1210
1220
1230
1240
1250

CCCGGCGTGCAGGGCAAGAAGGTCCGCAAGCCCAGCAGCAAAGGCCTGGC 
GGGCCGCACGTCCCGTTCTTCCAGGCGTTCGGGTCGTCGTTTCCGGACCG $\begin{array}{lllllllllllllllll}P & G & V & Q & G & K & K & V & R & K & P & S & S & K & G & L & A>\end{array}$ TRANSLATION OF ICN1 [A]

$\begin{array}{lllll}1260 & 1270 & 1280 & 1290 & 1300\end{array}$

CTGTGGAAGCAAGGAGGCCAAGGACCTCAAGGCACGGAGGAAGAAGTCCC

GACACCTTCGTTCCTCCGGTTCCTGGAGTTCCGTGCCTCCTTCTTCAGGG

$\begin{array}{llllllllllllllll}C & G & S & K & E & A & K & D & L & K & A & R & R & K & K & S>\end{array}$ TRANSLATION OF ICN1 [A]
1310
1320
1330
1340
1350

AGGATGGCAAGGGCTGCCTGCTGGACAGCTCCGGCATGCTCTCGCCCGTG TCCTACCGTTCCCGACGGACGACCTGTCGAGGCCGTACGAGAGCGGGCAC

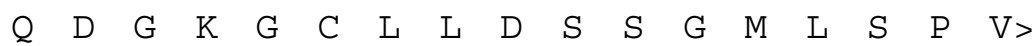
TRANSLATION OF ICN1 [A]
1360
1370
1380
1390
1400

GACTCCCTGGAGTCACCCCATGGCTACCTGTCAGACGTGGCCTCGCCGCC CTGAGGGACCTCAGTGGGGTACCGATGGACAGTCTGCACCGGAGCGGCGG $\begin{array}{lllllllllllllllll}D & S & L & E & S & P & H & G & Y & L & S & D & V & A & S & P & P>\end{array}$ TRANSLATION OF ICN1 [A]
1410
1420
1430
1440
1450

ACTGCTGCCCTCCCCGTTCCAGCAGTCTCCGTCCGTGCCCCTCAACCACC TGACGACGGGAGGGGCAAGGTCGTCAGAGGCAGGCACGGGGAGTTGGTGG

$$
\begin{array}{llllllllllllllll}
L & L & P & S & P & F & Q & Q & S & P & S & V & P & L & N & H>
\end{array}
$$
TRANSLATION OF ICN1 [A]
1460
1470
1480
1490
1500

TGCCTGGGATGCCCGACACCCACCTGGGCATCGGGCACCTGAACGTGGCG ACGGACCCTACGGGCTGTGGGTGGACCCGTAGCCCGTGGACTTGCACCGC $\begin{array}{lllllllllllllllll}L & P & G & M & P & D & T & H & L & G & I & G & H & L & N & V & A>\end{array}$ TRANSLATION OF ICN1 [A]

$\begin{array}{lllll}1510 & 1520 & 1530 & 1540 & 1550\end{array}$

GCCAAGCCCGAGATGGCGGCGCTGGGTGGGGGCGGCCGGCTGGCCTTTGA CGGTTCGGGCTCTACCGCCGCGACCCACCCCCGCCGGCCGACCGGAAACT $\begin{array}{lllllllllllllllll}A & K & P & E & M & A & A & L & G & G & G & G & R & L & A & F & E>\end{array}$ TRANSLATION OF ICN1 [A]

$$
\begin{array}{lllll}
1560 & 1570 & 1580 & 1590 & 1600
\end{array}
$$

GACTGGCCCACCTCGTCTCTCCCACCTGCCTGTGGCCTCTGGCACCAGCA CTGACCGGGTGGAGCAGAGAGGGTGGACGGACACCGGAGACCGTGGTCGT

$\begin{array}{llllllllllllllll}T & G & P & P & R & L & S & H & L & P & V & A & S & G & T & S>\end{array}$ TRANSLATION OF ICN1 [A]

$\begin{array}{lllll}1610 & 1620 & 1630 & 1640 & 1650\end{array}$

CCGTCCTGGGCTCCAGCAGCGGAGGGGCCCTGAATTTCACTGTGGGCGGG GGCAGGACCCGAGGTCGTCGCCTCCCCGGGACTTAAAGTGACACCCGCCC $\begin{array}{llllllllllllllllll}T & V & L & G & S & S & S & G & G & A & L & N & F & T & V & G & G>\end{array}$ TRANSLATION OF ICN1 [A]

$$
\begin{array}{lllll}
1660 & 1670 & 1680 & 1690 & 1700
\end{array}
$$

TCCACCAGTTTGAATGGTCAATGCGAGTGGCTGTCCCGGCTGCAGAGCGG 
AGGTGGTCAAACTTACCAGTTACGCTCACCGACAGGGCCGACGTCTCGCC $\begin{array}{lllllllllllllllll}S & T & S & L & N & G & Q & C & E & W & L & S & R & L & Q & S & G>\end{array}$ TRANSLATION OF ICN1 [A]
1710
1720
1730
1740
1750

CATGGTGCCGAACCAATACAACCCTCTGCGGGGGAGTGTGGCACCAGGCC GTACCACGGCTTGGTTATGTTGGGAGACGCCCCCTCACACCGTGGTCCGG

$\begin{array}{llllllllllllllll}M & V & P & N & Q & Y & N & P & L & R & G & S & V & A & P & G>\end{array}$ TRANSLATION OF ICN1 [A]
1760
1770
1780
1790
1800

CCCTGAGCACACAGGCCCCCTCCCTGCAGCATGGCATGGTAGGCCCGCTG GGGACTCGTGTGTCCGGGGGAGGGACGTCGTACCGTACCATCCGGGCGAC $\begin{array}{llllllllllllllllll}P & L & S & T & Q & A & P & S & L & Q & H & G & M & V & G & P & L>\end{array}$ TRANSLATION OF ICN1 [A]
1810
1820
1830
1840
1850

CACAGTAGCCTTGCTGCCAGCGCCCTGTCCCAGATGATGAGCTACCAGGG GTGTCATCGGAACGACGGTCGCGGGACAGGGTCTACTACTCGATGGTCCC $\begin{array}{lllllllllllllllll}H & S & S & L & A & A & S & A & L & S & Q & M & M & S & Y & Q & G>\end{array}$ TRANSLATION OF ICN1 [A]

$\begin{array}{lllll}1860 & 1870 & 1880 & 1890 & 1900\end{array}$

CCTGCCCAGCACCCGGCTGGCCACCCAGCCTCACCTGGTGCAGACCCAGC GGACGGGTCGTGGGCCGACCGGTGGGTCGGAGTGGACCACGTCTGGGTCG

$\begin{array}{llllllllllllllllllll}L & P & S & T & R & L & A & T & Q & P & H & L & V & Q & T & Q>\end{array}$ TRANSLATION OF ICN1 [A]
1910
1920
1930
1940
1950

AGGTGCAGCCACAAAACTTACAGATGCAGCAGCAGAACCTGCAGCCAGCA TCCACGTCGGTGTTTTGAATGTCTACGTCGTCGTCTTGGACGTCGGTCGT $\begin{array}{lllllllllllllllll}Q & V & Q & P & Q & N & L & Q & M & Q & Q & Q & N & L & Q & P & A>\end{array}$ TRANSLATION OF ICN1 [A]
1960
1970
1980
1990
2000

AACATCCAGCAGCAGCAAAGCCTGCAGCCGCCACCACCACCACCACAGCC TTGTAGGTCGTCGTCGTTTCGGACGTCGGCGGTGGTGGTGGTGGTGTCGG $\begin{array}{llllllllllllllllll}N & I & Q & Q & Q & Q & S & L & Q & P & P & P & P & P & P & Q & P>\end{array}$ TRANSLATION OF ICN1 [A]

$2010 \quad 2020 \quad 2030 \quad 2040 \quad 2050$

GCACCTTGGCGTGAGCTCAGCAGCCAGCGGCCACCTGGGCCGGAGCTTCC CGTGGAACCGCACTCGAGTCGTCGGTCGCCGGTGGACCCGGCCTCGAAGG

$\begin{array}{llllllllllllllll}H & L & G & V & S & S & A & A & S & G & H & L & G & R & S & F>\end{array}$ TRANSLATION OF ICN1 [A]

$2060 \quad 2070 \quad 2080 \quad 2090 \quad 2100$

TGAGTGGAGAGCCGAGCCAGGCAGACGTGCAGCCACTGGGCCCCAGCAGC ACTCACCTCTCGGCTCGGTCCGTCTGCACGTCGGTGACCCGGGGTCGTCG $\begin{array}{lllllllllllllllllllllllllll}L & S & G & E & P & S & Q & A & D & V & Q & P & L & G & P & S & S>\end{array}$ TRANSLATION OF ICN1 [A]

$$
2110 \quad 2120 \quad 2130 \quad 2140 \quad 2150
$$

CTGGCGGTGCACACTATTCTGCCCCAGGAGAGCCCCGCCCTGCCCACGTC 


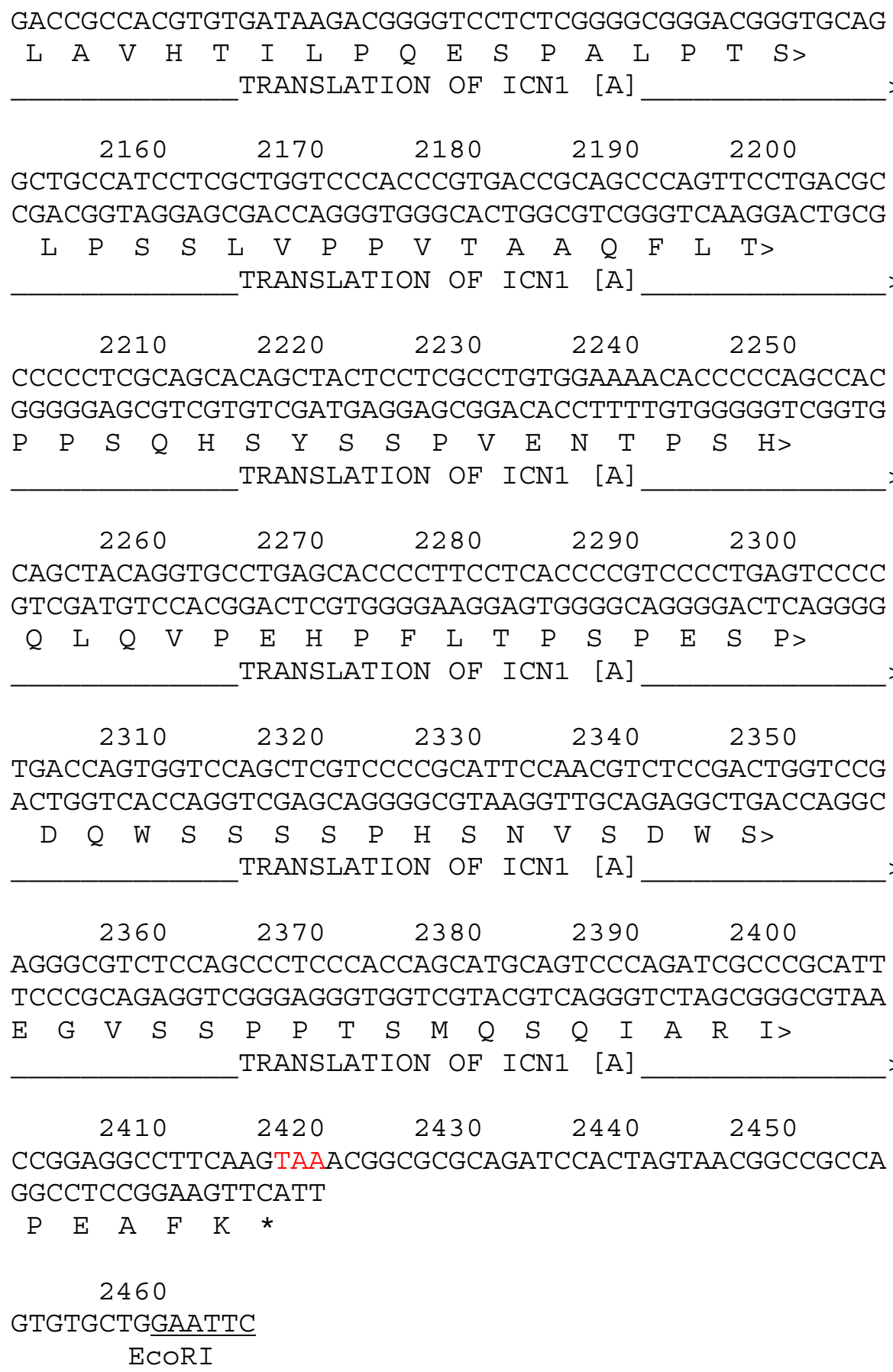

$2410 \quad 2420 \quad 2430 \quad 2440 \quad 2450$

CCGGAGGCCTTCAAGTAAACGGCGCGCAGATCCACTAGTAACGGCCGCCA GGCCTCCGGAAGTTCATT

For C-terminal tags 3'- end of ICN1 was modified by deletion of a single "C" nucleotide to create an AflII site next to TAA - STOP codone: ...CTTcAAGtaa -> CTTAAGtaa. Then -HA or - 6xHis sequences were added as follows: -HA sequence:

AflII XhoI

CTTAAGTTACCCATACGATGTTCCTGACTATGCGTAACTCGAG

$\begin{array}{llllllllllll}L & S & Y & P & Y & D & V & P & D & Y & A & \text { stop }\end{array}$

$-6 \mathrm{xHis}$ sequence:

AflII

XhoI

CTTAAGTCACCACCACCACCACCACTGACTCGAG

$\begin{array}{llllllllll}L & S & H & H & H & H & H & H & \text { Stop }\end{array}$ 
2) Recombinant CSL gene.

$10 \quad 20 \quad 30 \quad 40 \quad 50$

ATGGACTACAAAGACGATGACGACAAGCTTATGGACCACACGGAGGGC

TACCTGATGTTTCTGCTACTGCTGTTCGAATACCTGGTGTGCCTCCCG

$\begin{array}{llllllllllllllll}M & D & Y & K & D & D & D & D & K & L & M & D & H & T & E & G>\end{array}$

TRANSLATION OF FLAGCSLRECOMBINANT [A]

$\begin{array}{lllll}60 & 70 & 80 & 90 & 100\end{array}$

TTGCCCGCGGAGGAGCCGCCTGCGCATGCTCCATCGCCTGGGAAATTTGG AACGGGCGCCTCCTCGGCGGACGCGTACGAGGTAGCGGACCCTTTAAACC

$\begin{array}{lllllllllllllllll}L & P & A & E & E & P & P & A & H & A & P & S & P & G & K & F & G>\end{array}$ TRANSLATION OF FLAGCSLRECOMBINANT [A]

$\begin{array}{lllll}110 & 120 & 130 & 140 & 150\end{array}$

TGAGCGGCCTCCACCTAAACGACTTACTAGGGAAGCTATGCGAAATTATT

ACTCGCCGGAGGTGGATTTGCTGAATGATCCCTTCGATACGCTTTAATAA

$\begin{array}{llllllllllllllll}E & R & P & P & P & K & R & L & T & R & E & A & M & R & N & Y>\end{array}$ TRANSLATION OF FLAGCSLRECOMBINANT [A]
160
170
180
190
200

TAAAAGAGCGAGGGGATCAAACAGTACTTATTCTTCATGCAAAAGTTGCA ATTTTCTCGCTCCCCTAGTTTGTCATGAATAAGAAGTACGTTTTCAACGT

$\begin{array}{lllllllllllllllll}L & K & E & R & G & D & Q & T & V & L & I & L & H & A & K & V & A>\end{array}$ TRANSLATION OF FLAGCSLRECOMBINANT [A]
210
220
230
240
250

CAGAAGTCATATGGAAATGAAAAAAGGTTTTTTTGCCCACCTCCTTGTGT GTCTTCAGTATACCTTTACTTTTTTCCAAAAAAACGGGTGGAGGAACACA

$\begin{array}{llllllllllllllllll}Q & K & S & Y & G & N & E & K & R & F & F & C & P & P & P & C & V>\end{array}$ TRANSLATION OF FLAGCSLRECOMBINANT [A]

$260 \quad 270 \quad 280 \quad 290 \quad 300$

ATATCTTATGGGCAGCGGATGGAAGAAAAAAAAAGAACAAATGGAACGCG

TATAGAATACCCGTCGCCTACCTTCTTTTTTTTTCTTGTTTACCTTGCGC

$\begin{array}{lllllllllllllllll}Y & L & M & G & S & G & W & K & K & K & K & E & Q & M & E & R>\end{array}$ TRANSLATION OF FLAGCSLRECOMBINANT [A]
310
320
330
340
350

ATGGTTGTTCTGAACAAGAGTCTCAACCGTGTGCATTTATTGGGATAGGA TACCAACAAGACTTGTTCTCAGAGTTGGCACACGTAAATAACCCTATCCT

$\begin{array}{lllllllllllllllll}D & G & C & S & E & Q & E & S & Q & P & C & A & F & I & G & I & G>\end{array}$ TRANSLATION OF FLAGCSLRECOMBINANT [A]

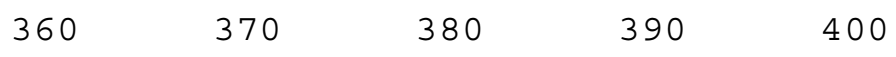

AATAGTGACCAAGAAATGCAGCAGCTAAACTTGGAAGGAAAGAACTATTG TTATCACTGGTTCTTTACGTCGTCGATTTGAACCTTCCTTTCTTGATAAC $\begin{array}{lllllllllllllllll}N & S & D & Q & E & M & Q & Q & L & N & L & E & G & K & N & Y & C>\end{array}$ TRANSLATION OF FLAGCSLRECOMBINANT [A]

$$
\begin{array}{llll}
410 & 420 & 430 & 440
\end{array} 450
$$

CACAGCCAAAACATTGTATATATCTGACTCAGACAAGCGAAAGCACTTCA GTGTCGGTTTTGTAACATATATAGACTGAGTCTGTTCGCTTTCGTGAAGT

$\begin{array}{llllllllllllllll}T & A & K & T & L & Y & I & S & D & S & D & K & R & K & H & F>\end{array}$ TRANSLATION OF FLAGCSLRECOMBINANT [A] 
$460 \quad 470 \quad 480 \quad 490 \quad 500$

TTTTTTCTGTAAAGATGTTCTATGGCAACAGTGATGACATTGGTGTGTTC AAAAAAGACATTTCTACAAGATACCGTTGTCACTACTGTAACCACACAAG $\begin{array}{lllllllllllllllll}I & F & S & V & K & M & F & Y & G & N & S & D & D & I & G & V & F>\end{array}$ TRANSLATION OF FLAGCSLRECOMBINANT [A]
510
520
530
540
550

CTCAGCAAGCGGATAAAAGTCATCTCCAAACCTTCCAAAAAGAAGCAGTC GAGTCGTTCGCCTATTTTCAGTAGAGGTTTGGAAGGTTTTTCTTCGTCAG

$\begin{array}{lllllllllllllllll}L & S & K & R & I & K & V & I & S & K & P & S & K & K & K & Q & S>\end{array}$ TRANSLATION OF FLAGCSLRECOMBINANT [A]
560
570
580
590
600

ATTGAAAAATGCTGACTTATGCATTGCCTCAGGAACAAAGGTGGCTCTGT TAACTTTTTACGACTGAATACGTAACGGAGTCCTTGTTTCCACCGAGACA

$\begin{array}{llllllllllllllll}L & K & N & A & D & L & C & I & A & S & G & T & K & V & A & L>\end{array}$ TRANSLATION OF FLAGCSLRECOMBINANT [A]
610
620
630
640
650

TTAATCGACTACGATCCCAGACAGTTAGTACCAGATACTTGCATGTAGAA AATTAGCTGATGCTAGGGTCTGTCAATCATGGTCTATGAACGTACATCTT $\begin{array}{llllllllllllllllll}F & N & R & L & R & S & Q & T & V & S & T & R & Y & L & H & V & E>\end{array}$ TRANSLATION OF FLAGCSLRECOMBINANT [A]
660
670
680
690
700

GGAGGTAATTTTCATGCCAGTTCACAGCAGTGGGGAGCCTTTTTTATTCA CCTCCATTAAAAGTACGGTCAAGTGTCGTCACCCCTCGGAAAAAATAAGT $\begin{array}{llllllllllllllllll}G & G & N & F & H & A & S & S & Q & Q & W & G & A & F & F & I & H>\end{array}$ TRANSLATION OF FLAGCSLRECOMBINANT [A]
710
720
730
740
750

TCTCTTGGATGATGATGAATCAGAAGGAGAAGAATTCACAGTCCGAGATG AGAGAACCTACTACTACTTAGTCTTCCTCTTCTTAAGTGTCAGGCTCTAC

$\begin{array}{llllllllllllllll}L & L & D & D & D & E & S & E & G & E & E & F & T & V & R & D>\end{array}$ TRANSLATION OF FLAGCSLRECOMBINANT [A]
760
770
780
790
800

TCTACATCCATTATGGACAAACATGCAAACTTGTGTGCTCAGTTACTGGC AGATGTAGGTAATACCTGTTTGTACGTTTGAACACACGAGTCAATGACCG $\begin{array}{lllllllllllllllll}V & Y & I & H & Y & G & Q & T & C & K & L & V & C & S & V & T & G>\end{array}$ TRANSLATION OF FLAGCSLRECOMBINANT [A]
810
820
830
840
850

ATGGCACTCCCAAGATTGATAATTATGAAAGTTGATAAGCATACCGCATT TACCGTGAGGGTTCTAACTATTAATACTTTCAACTATTCGTATGGCGTAA $\begin{array}{lllllllllllllllll}M & A & L & P & R & L & I & I & M & K & V & D & K & H & T & A & L>\end{array}$ TRANSLATION OF FLAGCSLRECOMBINANT [A]
860
870
880
890
900

ATTGGATGCAGATGATCCTGTGTCACAACTCCATAAATGTGCATTTTACC TAACCTACGTCTACTAGGACACAGTGTTGAGGTATTTACACGTAAAATGG

$\begin{array}{llllllllllllllll}L & D & A & D & D & P & V & S & Q & L & H & K & C & A & F & Y>\end{array}$ TRANSLATION OF FLAGCSLRECOMBINANT [A] 
$910 \quad 920 \quad 930 \quad 940 \quad 950$

TTAAGGATACAGAAAGAATGTATTTGTGCCTTTCTCAAGAAAGAATAATT AATTCCTATGTCTTTCTTACATAAACACGGAAAGAGTTCTTTCTTATTAA $\begin{array}{llllllllllllllllll}L & K & D & T & E & R & M & Y & L & C & L & S & Q & E & R & I & I>\end{array}$ TRANSLATION OF FLAGCSLRECOMBINANT [A]
960
970
980
990
1000

CAATTTCAGGCCACTCCATGTCCAAAAGAACCAAATAAAGAGATGATAAA GTTAAAGTCCGGTGAGGTACAGGTTTTCTTGGTTTATTTCTCTACTATTT

$\begin{array}{llllllllllllllllll}Q & F & Q & A & T & P & C & P & K & E & P & N & K & E & M & I & N>\end{array}$ TRANSLATION OF FLAGCSLRECOMBINANT [A]
1010
1020
1030
1040
1050

TGATGGCGCTTCCTGGACAATCATTAGCACAGATAAGGCAGAGTATACAT ACTACCGCGAAGGACCTGTTAGTAATCGTGTCTATTCCGTCTCATATGTA

$\begin{array}{llllllllllllllll}D & G & A & S & W & T & I & I & S & T & D & K & A & E & Y & T>\end{array}$ TRANSLATION OF FLAGCSLRECOMBINANT [A]
1060
1070
1080
1090
1100

TTTATGAGGGAATGGGCCCTGTCCTTGCCCCAGTCACTCCTGTGCCTGTG AAATACTCCCTTACCCGGGACAGGAACGGGGTCAGTGAGGACACGGACAC $\begin{array}{llllllllllllllllll}F & Y & E & G & M & G & P & V & L & A & P & V & T & P & V & P & V>\end{array}$ TRANSLATION OF FLAGCSLRECOMBINANT [A]
1110
1120
1130
1140
1150

GTAGAGAGCCTTCAGTTGAATGGCGGTGGGGACGTAGCAATGCTTGAACT CATCTCTCGGAAGTCAACTTACCGCCACCCCTGCATCGTTACGAACTTGA $\begin{array}{llllllllllllllllll}V & E & S & L & Q & L & N & G & G & G & D & V & A & M & L & E & L>\end{array}$ TRANSLATION OF FLAGCSLRECOMBINANT [A]
1160
1170
1180
1190
1200

TACAGGACAGAATTTCACTCCAAATTTACGAGTGTGGTTTGGGGATGTAG ATGTCCTGTCTTAAAGTGAGGTTTAAATGCTCACACCAAACCCCTACATC

$\begin{array}{llllllllllllllll}T & G & Q & N & F & T & P & N & L & R & V & W & F & G & D & V>\end{array}$ TRANSLATION OF FLAGCSLRECOMBINANT [A]
1210
1220
1230
1240
1250

AAGCTGAAACTATGTACAGGTGTGGAGAGAGTATGCTCTGTGTCGTCCCA TTCGACTTTGATACATGTCCACACCTCTCTCATACGAGACACAGCAGGGT $\begin{array}{llllllllllllllllll} & \mathrm{E} & \mathrm{A} & \mathrm{E} & \mathrm{T} & \mathrm{M} & \mathrm{Y} & \mathrm{R} & \mathrm{C} & \mathrm{G} & \mathrm{E} & \mathrm{S} & \mathrm{M} & \mathrm{L} & \mathrm{C} & \mathrm{V} & \mathrm{V} & \mathrm{P}>\end{array}$ TRANSLATION OF FLAGCSLRECOMBINANT [A]
1260
1270
1280
1290
1300

GACATTTCTGCATTCCGAGAAGGTTGGAGATGGGTCCGGCAACCAGTCCA CTGTAAAGACGTAAGGCTCTTCCAACCTCTACCCAGGCCGTTGGTCAGGT

$$
\begin{array}{lllllllllllllllll}
D & I & S & A & F & R & E & G & W & R & W & V & R & Q & P & V & Q>
\end{array}
$$
TRANSLATION OF FLAGCSLRECOMBINANT [A]
1310
1320
1330
1340
1350

GGTTCCAGTAACTTTGGTCCGAAATGATGGAATCATTTATTCCACCAGCC CCAAGGTCATTGAAACCAGGCTTTACTACCTTAGTAAATAAGGTGGTCGG

$\begin{array}{llllllllllllllll}V & P & V & T & L & V & R & N & D & G & I & I & Y & S & T & S>\end{array}$ TRANSLATION OF FLAGCSLRECOMBINANT [A] 


$$
1360 \quad 1370 \quad 1380 \quad 1390 \quad 1400
$$

TTACCTTTACCTACACACCAGAACCAGGGCCACGGCCACATTGCAGTGTA AATGGAAATGGATGTGTGGTCTTGGTCCCGGTGCCGGTGTAACGTCACAT $\begin{array}{lllllllllllllllll}L & T & F & T & Y & T & P & E & P & G & P & R & P & H & C & S & V>\end{array}$ TRANSLATION OF FLAGCSLRECOMBINANT [A]
1410
1420
1430
1440
1450

GCAGGAGCAATCCTTCCAGCCAATTCAAGCCAGGTGCCCCCTAACGAATC CGTCCTCGTTAGGAAGGTCGGTTAAGTTCGGTCCACGGGGGATTGCTTAG
$\begin{array}{lllllllllllllllll}A & G & A & I & L & P & A & N & S & S & Q & V & P & P & N & E & S>\end{array}$ TRANSLATION OF FLAGCSLRECOMBINANT [A]

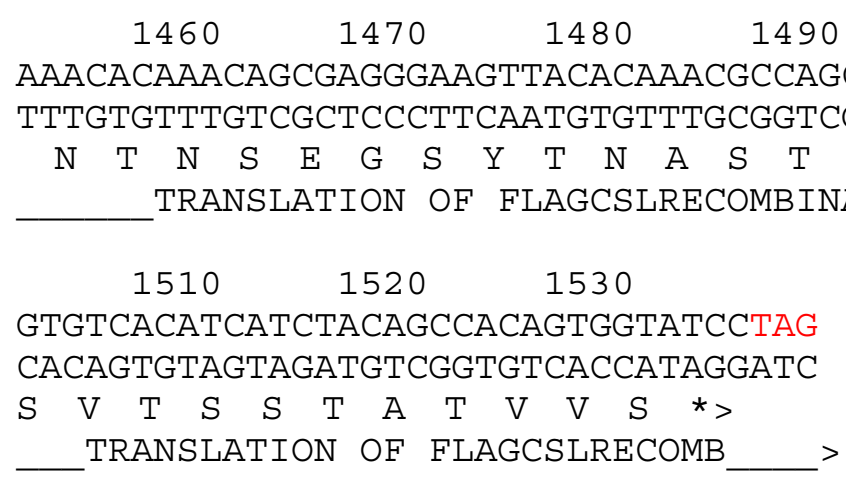

For 6xHis-tag expression the last six nucleotides of CSL sequence were modified by mutagenesis to create XhoI site: TCCTAG -> CTCGAG. The modification allowed cloning directly in pET28a vector between 5' - NcoI and 3' - XhoI sites in frame with 3'- 6xHis-tag sequence, provided in the vector.

3) Quantification Tables 1 and 2 for EMSA.

Table 1. Quantification of ${ }^{32}$ P-activity in EMSA bands with bacterially expressed/purified CSL and ICN1, 6 independent experiments. In bold-data correspondent to EMSA in Figure 3.

\begin{tabular}{cccccc}
\hline & 59 bp CPS probe, No ICN1, pxl & & & 59 bp CPS probe, + ICN, pxl \\
\hline DNA-CSL & DNA & Total & DNA-CSL & DNA & Total \\
$\mathbf{3 0 6 7 5 8}$ & $\mathbf{9 5 8 6 6}$ & $\mathbf{4 0 2 6 2 4}$ & $\mathbf{1 4 6 7 8 2}$ & $\mathbf{3 1 9 0 4 5}$ & $\mathbf{4 6 5 8 2 7}$ \\
148573 & 434698 & 583271 & 64652 & 598300 & 662952 \\
136546 & 160695 & 297241 & 92470 & 196675 & 289145 \\
24636 & 17709 & 42345 & 7829 & 29633 & 37462 \\
39090 & 76385 & 115475 & 32326 & 82371 & 114697 \\
39745 & 56339 & 96084 & 34521 & 61949 & 96570 \\
\hline
\end{tabular}

${ }^{1}$ Pxl units = amount of radioactivity converted to pixels by phosphorimaging.

Table 2. Quantification of ${ }^{32} \mathrm{P}$-activity in EMSA bands with CSL/HEK293T and ICN1/HEK293T extracts, 7 independent experiments. In bold-data correspondent to EMSA in Figure 6.

\begin{tabular}{ccccccc}
\hline & No ICN1/HEK293T, pxl & \multicolumn{5}{c}{ + ICN1/HEK293T, pxl } \\
\hline DNA-CSL & DNA & Total & Shift & DNA & Non-specific & Total \\
24636 & 17709 & 42345 & 25512 & 14187 & 3205 & 42904 \\
$\mathbf{3 9 0 9 0}$ & $\mathbf{7 6 3 8 5}$ & $\mathbf{1 1 5 4 7 5}$ & $\mathbf{4 7 8 6 3}$ & $\mathbf{6 0 4 8 9}$ & $\mathbf{1 1 1 0 7}$ & $\mathbf{1 1 9 4 5 9}$ \\
39745 & 56339 & 96084 & 33145 & 52297 & 9245 & 94687 \\
53628 & 117939 & 171567 & 53964 & 102001 & 9136 & 165101 \\
157099 & 268372 & 425471 & 160484 & 228974 & 32693 & 422151 \\
167115 & 255395 & 422510 & 182137 & 232023 & 1053 & 414160 \\
120387 & 126638 & 247025 & 170213 & 114081 & 978 & 284294 \\
\hline
\end{tabular}

